\title{
50. NEOGENE PALEOCLIMATE DEVELOPMENT OF THE ANTARCTIC WEDDELL SEA REGION: ORGANIC GEOCHEMISTRY1
}

\author{
Stephen A. Macko² and Christopher P. G. Pereira ${ }^{3}$
}

\begin{abstract}
Stable carbon and nitrogen isotopic compositions as well as organic carbon and total nitrogen contents of cored material are reported for the Weddell Sea, Ocean Drilling Program (ODP) Sites 689 and 690 (Maud Rise), Site 693 (continental margin), and Site 694 (abyssal plain). Results from both high resolution sampling (up to six samples per section) and low resolution (one sample per section) are documented. In general, these results indicate large changes in the types and amounts of carbon and nitrogen preserved in the sediments of the Weddell Sea region during the past 25 m.y., with an especially important and dramatic event coinciding with the western Antarctic ice-sheet becoming a semi-permanent or permanent feature about $5 \mathrm{Ma}$.

The overall results may be correlated with the onset of major ice-sheets on West Antarctica, stabilization of the icesheet in the Pliocene and the intensified recycling of organic carbon and total nitrogen, which is possibly the result of increased ice cover. Evidence is also presented for either low production of organic carbon or the presence of a water column, in the eastern Weddell Sea during the early and middle Neogene, which was highly corrosive to organic matter. This condition, together with slow sediment accumulation rates, inhibited the preservation of carbon in the sediments.
\end{abstract}

\section{INTRODUCTION}

Holes were drilled at nine separate sites during Ocean Drilling Program (ODP) Leg 113 in the Weddell Sea, in order to yield information concerning: (1) the initiation of the formation of the Antarctic ice-sheets, and their permanency; (2) the variations in Antarctic Bottom Water as a result of cryospheric development; and (3) the evolution and history of primary productivity in the region and its linkages to climate. This paper reports on the results of stable carbon and nitrogen isotopic measurements and organic content analyses for samples collected at four sites from Leg 113 representing three distinct environments (Fig. 1).

The Maud Rise sites are located on the crest of the Rise in $2083 \mathrm{~m}$ of water (Site 689), and on the flank in $2914 \mathrm{~m}$ of water (Site 690). Holes were drilled at these sites, which are isolated from continental effects, to yield information on open ocean environments. Site 693, in $2359 \mathrm{~m}$ of water, on the East Antarctic continental margin, was drilled to investigate terrigenous influences on the Weddell Sea. Holes were drilled at Site 694, in the Weddell Sea basin at $4653 \mathrm{~m}$ to examine both the history of bottom water production, particularly the origin of the present day Weddell Sea Bottom Water and, through study of the distal turbidites, to establish a record of continental glaciation and pre-glacial vegetation.

This present study also sought to observe relationships between sedimentology and organic geochemical measurements, and relate those findings to productivity and changes in source materials, or variations in the biota and water masses influencing the sediment. Because of powerful feedback mechanisms which are related to ice albedo and deep-water formation, these results could aid in the understanding of glacial evolution in Antarctica as well as in the high Arctic (observed during ODP

\footnotetext{
${ }^{1}$ Barker, P. F., Kennett, J. P., et al., 1990. Proc. ODP, Sci., Results. 113: College Station, TX (Ocean Drilling Program).

2 Department of Earth Sciences, Memorial University, St. John's, Newfoundland, Canada A1B 3 X5.

3 Department of Earth Sciences, Memorial University, St John's, Newfoundland, Canada A1B 3X5.
}

Leg 105; Macko, 1989), and could also be important in the evaluation of climatic change.

The organic contents and the isotopic compositions of sediments have been used to suggest the extent and timing of glacial influences in Quaternary marine sequences. These studies are based on the premise that the organic material found in sediments is reflective of the many biological and chemical transformations of the original source carbon or nitrogen. That organic material may have originated in the marine photic zone, or in nearshore environments as terrigenous debris, or more likely, as a mixture of the two. This study makes use of organic content and the stable isotopic compositions of carbon and nitrogen in the sediments to aid in the delineation of the origins and amounts of organics in the water column of the Weddell Sea.

In numerous marine environments, relative contributions of terrigenous and marine inputs have been documented through the use of stable carbon isotopes. For example, in the Gulf of Mexico, surficial sediments contain increasing amounts of the heavier isotope of carbon $\left({ }^{13} \mathrm{C}\right.$ ) with increasing distance from land, and yield evidence for decreasing influence of terrigenous carbon influences (Sackett and Thompson, 1963; Hedges and Parker, 1976; Gearing et al., 1977). In the deltas of both the Pedernales in Venezuela (Eckelman et al, 1962) and Niger rivers (Gearing et al., 1977) woody fragments and more finely disseminated terrestrial plant debris give a clear terrigenous isotopic signature to deltaic sediments. In more northern Arctic environments, the transport of terrigenous material may be more extensive. In the Beaufort Sea, lateral transport of terrestrial debris is enhanced by ice-rafting (Gearing et al., 1977). These variations in source are recorded in the sediments of an area. In the Gulf of Mexico, variations downcore have been correlated with glacial and interglacial episodes which are related to sea-level lowering and the changing influence of the Mississippi River (Parker et al., 1972; Newman et al., 1973). In systems which are exclusively or heavily dominated by marine productivity, other environmental parameters such as temperature, growth rate, species distribution, and $\mathrm{CO}_{2}$ availability may affect carbon isotopic composition (Sackett et al., 1965; Degens et al., 1968; Gearing et al., 1977; Fontugne and Duplessy, 1978; 1981; Degens, 1969). In Baffin Bay and the Labrador Sea, large-scale changes in $\delta^{13} \mathrm{C}$ were interpreted to be strongly influenced by changes in marine 


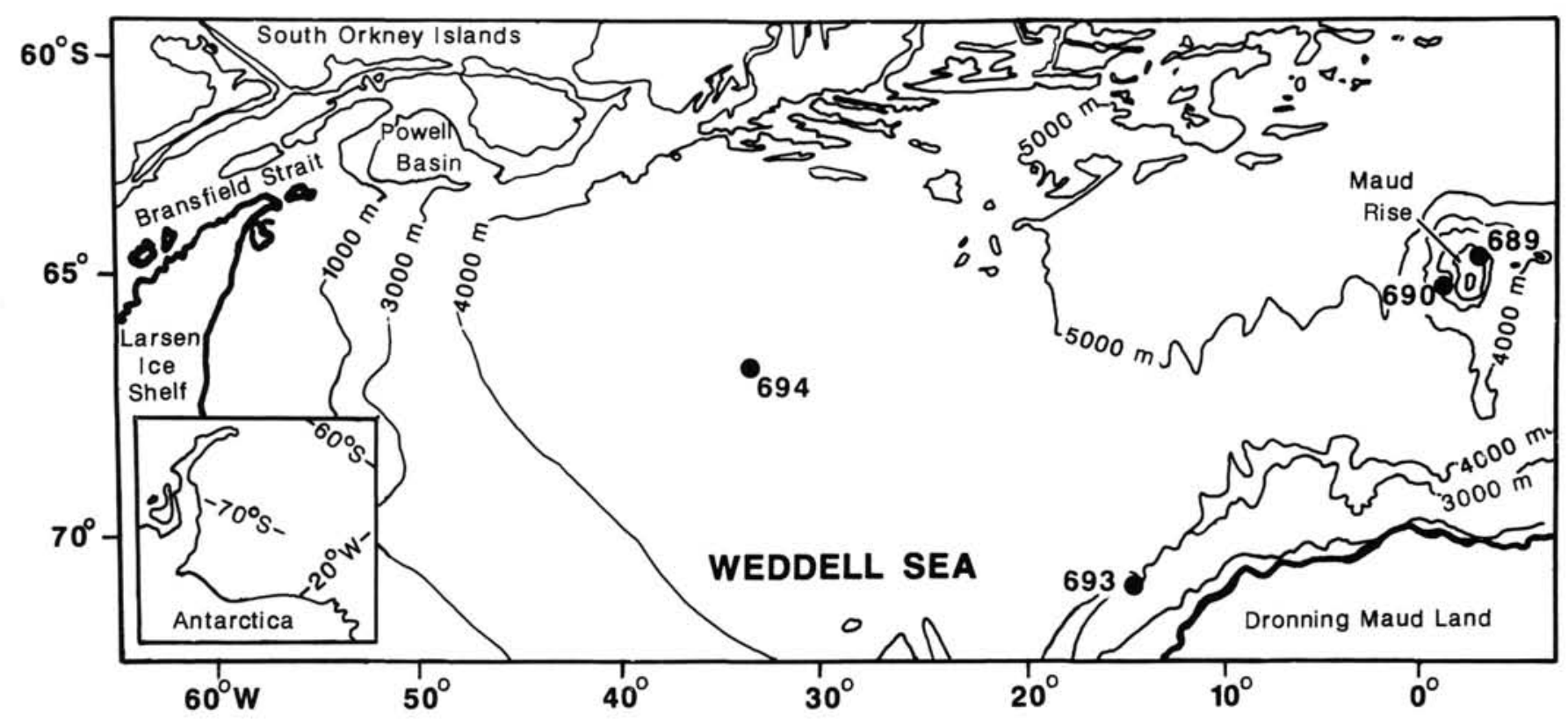

Figure 1. Map showing locations of Leg 113 Sites in this study.

planktonic productivity, and by the effect of ice-rafted debris on the overall accumulation of organic matter in sediments of the region (Macko, 1989).

Cooler temperatures in water masses have been correlated with planktonic organic material which is depleted in ${ }^{13} \mathrm{C}$. This observation and interpretation is based on the premise that the isotopic fractionation by phytoplankton growing in the open ocean will be affected by temperature. These temperature-induced signatures have been interpreted to be the cause for carbon isotopic variations observed in high latitude deep-sea sediments (Sackett, 1986a; 1986b; Sackett et al., 1974; Rogers et al., 1972). However, carbon isotopic variations may also be associated with changing populations of phytoplankton. For example, in the Antarctic, the transition of a diatom ooze to a dinoflagellate/coccolithophore dominated sediment at the Antarctic Convergence is associated with a decrease in the carbon isotopic signature (Sackett et al., 1974). Isotopic differences exist among phytoplankton of different size classes (hence different species) from the same bloom (Gearing et al., 1984). Such variability would certainly have impact on the organic isotopic record preserved in a sediment (Macko et al., 1987b).

In similar fashion, stable nitrogen isotopes may be useful indicators of the source of organic material entering the marine environment. Peters et al. (1978) correlated nitrogen isotope signatures with relative contributions of terrigenous and marine inputs into a sediment. Such contributions of organics can be quantified by simple mass balance calculations from which source estimates can be made (Macko 1981; 1983).

Because the major source of nitrogen utilized in terrestrial systems $\left(\mathrm{N}_{2}\right.$ via nitrogen fixation) is isotopically distinguishable from marine systems (oceanic nitrate through nitrate reduction), these sources may be resolved in many instances. Furthermore, in purely marine environments, the processes through which phytoplankton (or bacteria) incorporate nitrogen may be resolved if one can eliminate sources of nitrogen from land. The process of nitrogen fixation has a small isotopic fractionation associated with the utilization of molecular nitrogen. Such a process is easily distinguished from the more common mode of nitrate reduction in which phytoplankton fully utilize dissolved nitrate and reflect its isotopic signature (Macko et al., 1984). When algae are only able to use a small portion of the dissolved nitrate, more depleted ${ }^{15} \mathrm{~N}$ values may be evident, reflecting the isotopic fractionation associated with that incorporation (Macko et al., 1987a). Such changes may then be useful in the interpretation of the paleoceanographic record of an area. Sediments formed during periods of higher productivity, in which phytoplankton fully utilized the oceanic nitrate, may be more enriched in ${ }^{15} \mathrm{~N}$ than sediments associated with lower productivity in which larger fractionations are possible.

However, the situation is complicated by factors which include microbial action, diagenesis, and recycling of organics. Variations in isotopic signature may occur as a result of deamination reactions associated with organic rich materials (Sigleo and Macko, 1985; Zieman et al., 1984; Wada, 1980). Such processes of alteration may be especially important in changing isotopic signatures below the euphotic zone (Altabet and McCarthy, 1986).

\section{METHODS}

Sediment samples collected for organic content and isotopic composition were kept frozen until analysis. Samples were initially lyophilized and then acidified with $30 \% \mathrm{HCl}$ to remove carbonate. The carbonate-free residue was dried in order to preserve all soluble organic matter and porewater ammonium or nitrate. A portion of the dried material was then weighed and combusted in quartz for one hour at $850^{\circ} \mathrm{C}$ in the presence of purified cupric oxide wire and high purity granular copper, (Macko et al., 1984). The $\mathrm{N}_{2}$ and $\mathrm{CO}_{2}$ gases obtained were cryogenically isolated from other combustion products and analyzed on a V.G. Micromass PRISM stable isotope ratio mass spectrometer. On the basis of replicate analyses of samples, the reproducibility in combustion and measurement is within $\pm 0.2 \%$. Isotope data are presented as \%o:

$$
\delta^{\mathrm{N}} \mathrm{E}=\left[\frac{\mathrm{R}_{\text {sample }}}{\mathrm{R}_{\text {standard }}}-1\right] * 10^{3}
$$

where $\mathrm{N}$ is the isotope of the element $\mathrm{E}$ and $\mathrm{R}$ is the abundance ratio of the heavy to light isotope; the standard for ${ }^{15} \mathrm{~N}$ is atmospheric nitrogen and the standard for carbon is the Chicago 
PDB. For routine measurement, samples are analyzed versus a laboratory sub-standard tank of either pure nitrogen or carbon dioxide gas. Total nitrogen content was calculated from the ion intensity of the gas in a calibrated volume of the mass spectrometer; the organic carbon content was determined on a calibrated manometer in the vacuum purification line. Low resolution analysis would include one sample per $150 \mathrm{~cm}$ section while high resolution sampling could be as many as six samples per section. All data are presented in Table 1 in which the samples analyzed have been assigned Memorial University Newfoundland (MUN) numbers.

\section{RESULTS}

\section{Site 689}

At Site 689 , high and low resolution sampling was undertaken with a total of 319 samples being analyzed from Holes 689B, 689C, and 689D. Organic contents are generally low, less than $0.1 \%$ carbon and $0.05 \%$ total nitrogen, and exhibit a decreasing trend from the Paleocene to the Pleistocene with occasional erratic excursions from the low levels (Figs. 2, 3). The levels of organic carbon and total nitrogen average $0.06 \%$ and $0.04 \%$, respectively, and range from detection limits to $0.057 \%$ and detection limits to $0.38 \%$. The atomic ratio of carbon to nitrogen, $\mathrm{C} / \mathrm{N}$, shows more variation and ranges from 0.1 to 15.0 , with generally highest values during the Pliocene. Carbon isotopic values were typically $-27 \%$ during the Paleocene and Eocene, diminished to $-30 \%$ in the Oligocene and increased to values up to $-19 \%$ during the Pliocene and Pleistocene. The nitrogen isotopic $\left(\delta^{15} \mathrm{~N}\right)$ compositions show a general trend of decreasing from the Paleocene (approximately 7.8\%0) to the Pleistocene. The lowest values ${ }^{15} \mathrm{~N}(0.8 \%$ occur in samples from the Pliocene and Pleistocene. High resolution sampling for all types of analyses indicates that large excursions can occur within at least $0.1 \mathrm{~m}$, (representing 25,000 to $10,000 \mathrm{yr}$ at estimated sedimentation rates of 4-9 m/m.y.).

Significant correlations (Pearson $\mathrm{R}, \mathrm{p}=0.95$ ) were observed between nitrogen content and both carbon content and $\mathrm{C} / \mathrm{N}$, and between $\delta^{13} \mathrm{C}$ and either $\% \mathrm{C}, \% \mathrm{~N}, \mathrm{C} / \mathrm{N}$, or ${ }^{15} \mathrm{~N}$. The best $\mathrm{R}^{2}$ value for these correlations was 0.20 between $\% \mathrm{C}$ and $\% \mathrm{~N}$. The degrees of freedom used to determine significance levels were not corrected for any autocorrelation observed in these time series; with low $\mathrm{R}^{2}$ values, the correlations may be significant but are relatively weak.

\section{Site 690}

Ninety samples from Holes 690A and 690B were analyzed from this site (Figs. 4, 5). The trends observed on the crest of Maud Rise (Site 689) are generally similar to results from Site 690 on the flank. Organic contents are generally low with respective ranges and average compositions for carbon and total nitrogen being detection limit to $0.48 \%$ (average 0.09 ) and limit of detection to $0.56 \%$ (average $0.14 \%$ ). The carbon content is uniform with only a few excursions from low levels; nitrogen content is similar with the lowest values being recorded in the Pliocene-Pleistocene. The $\mathrm{C} / \mathrm{N}$ data for these samples are fairly uniform during the Oligocene and Miocene with more excursions from the average (1.8) value in the Pliocene-Pleistocene (total range $0.1-10.6$ ). The $\delta^{13} \mathrm{C}$ content of these samples was near $-27 \%$ during the Oligocene and Miocene, increased to $-20 \%$ in the Pliocene and decreases to $-24 \%$ in the Pleistocene. The nitrogen isotopic compositions of these samples were fairly uniform during the Oligocene and Miocene, near 5.5\%, and showed an increasing trend to $7.4 \%$ during the PliocenePleistocene. Sedimentation rates for this site are of a similar magnitude to those observed on the crest, resulting in a sam- pling time resolution of similar length $(25,000$ to $10,000 \mathrm{yr} / 0.1 \mathrm{~m}$ sampling).

Total nitrogen content is significantly correlated with $\% \mathrm{C}$, while $\delta^{13} \mathrm{C}$ variations correlate significantly with changes in $\% \mathrm{C}$ and $\mathrm{C} / \mathrm{N}$. The only correlations with $\mathrm{R}^{2}$ values greater than 0.1 are $\% \mathrm{C}$ with $\% \mathrm{~N}(0.50)$ and $\% \mathrm{C}$ with $\mathrm{C} / \mathrm{N}(0.37)$.

\section{Site 693}

The continental margin site was sampled 275 times for this study, with collections coming from Holes 693A and 693B. A distinct change is seen in all parameters in the Pliocene (approximate age of 5 m.y. using estimated sedimentation rates of 10 $\mathrm{m} / \mathrm{m}$.y. for the Pleistocene, $20 \mathrm{~m} / \mathrm{m}$.y. for the Pliocene, and 5 $\mathrm{m} / \mathrm{m} . \mathrm{y}$. for samples older than Pliocene in age; Figs. 6, 7). One sample, at $465 \mathrm{~m}$, of Cretaceous age, contained the greatest amount of carbon for the entire study. This amount was recorded to be $1.75 \%$; this sample also had six times as much nitrogen as the samples above it but it did not contain unusual ${ }^{15} \mathrm{~N}$ or ${ }^{13} \mathrm{C}$ compositions nor $\mathrm{C} / \mathrm{N}$. Generally, the carbon contents at this site are about $0.2 \%$ below the above mentioned 5 m.y. boundary, and decrease to approximately $0.1 \%$ above the boundary. Nitrogen contents are typically $0.05 \%$ below the 5 m.y. boundary, and drastically increase to $0.5 \%$ above that. The $\mathrm{C} / \mathrm{N}$ abundances average near six below the boundary and decline to less then one above the $5 \mathrm{~m} . \mathrm{y}$. depth. Nitrogen isotopic compositions at this site average approximately $4 \%$ during the Oligocene, increase to $6 \%$ in the early Miocene, and decline back to 3 to $4 \% 0$ in the late Miocene. From the Pliocene onward, the nitrogen isotopic values increase, with enrichments as high as $11.3 \%$ seen in the uppermost sediments of the Pleistocene. Carbon isotopic values of these sediments increase steadily from an average near $-25 \%$ in the Oligocene to the Miocene level of $-23 \%$. During the Pliocene-Pleistocene the compositions remain fairly constant near $-22 \%$.

The correlation matrix observed for the data collected on this site is similar to that seen at Sites 689 and 690. Carbon-13 significantly correlates with $\% \mathrm{C}, \% \mathrm{~N}, \mathrm{C} / \mathrm{N}$, and nitrogen isotopic levels. The organic carbon and total nitrogen contents significantly correlate with $\mathrm{C} / \mathrm{N}$, and have respective $\mathrm{R}^{2}$ values of 0.21 and 0.44 . As a consequence of the increased sedimentation rates in the Pliocene and Pleistocene, the minimum resolution in sampling decreases to between 10,000 and 5,000 $\mathrm{yr}$ in the high resolution samples from those periods.

\section{Site 694}

Recovery of sediments from Site 694 on the Weddell Sea abyssal plain was exceedingly poor. As a result, high resolution sampling was limited to a few short intervals in the Miocene section and one in the upper Pliocene (Figs. 8, 9). A total of 243 samples were analyzed from Holes 694A, 694B, and 694C. The levels of organic carbon preserved in the sediments were consistently higher than at all other sites in this study, with levels up to $1 \%$ being typical of the Miocene samples. The Pliocene samples average between $0.1 \%$ and $0.2 \%$. The total nitrogen found in these samples is low, ranging from detection limit to approximately $0.1 \%$ for all samples. Miocene samples had $\mathrm{C} / \mathrm{N}$ values which are fairly typical of marine sediments, between 10 and 15. The ratios decrease to five or less for the Pliocene age samples. The carbon and nitrogen isotopic compositions are relatively constant, with average values of $-22.9 \%$ and $4.0 \%$, respectively. Excursions to more depleted carbon and more enriched nitrogen compositions are occasionally seen in the Miocene samples. Sedimentation rates for the Pliocene are similar in magnitude to those seen at Site 693 and result in a sampling resolution of 10,000 to 5,000 yr. The Miocene sediments are likely the result of sediment slumping from the adjacent 
Table 1. ODP Leg 113: organic geochemistry results.

\begin{tabular}{|c|c|c|c|c|c|c|c|c|}
\hline Leg & Hole & Mun\# & Core & Depth & $\% \mathrm{C}$ & $\% \mathrm{~N}$ & $\mathrm{C} / \mathrm{N}$ & $\delta^{13} \mathrm{C}$ \\
\hline 113 & 689B & 1B3001 & $1 \mathrm{H} 01$ & 0.95 & 0.06 & 0.012 & 5.7 & -23.2 \\
\hline 113 & $689 \mathrm{~B}$ & IB3003 & IH03 & 3.01 & 0.06 & 0.013 & 5.5 & -26.9 \\
\hline 113 & 689B & 1B3004 & $1 \mathrm{H} 03$ & 3.42 & 0.06 & 0.012 & 5.8 & -25.8 \\
\hline 113 & 689B & 1B3005 & $1 \mathrm{H} 03$ & 4.05 & 0.04 & 0.010 & 4.8 & -22.9 \\
\hline 113 & 689B & 1B3006 & $1 \mathrm{H} 04$ & 4.60 & 0.04 & 0.105 & 0.4 & -23.9 \\
\hline 113 & 689B & 1B3008 & $2 \mathrm{H} 01$ & 5.49 & 0.02 & 0.024 & 1.1 & -25.9 \\
\hline 113 & 689B & 1B3009 & $2 \mathrm{H} 01$ & 6.73 & 0.09 & 0.105 & 1.0 & -25.8 \\
\hline 113 & 689B & 1B3010 & $2 \mathrm{H} 02$ & 6.84 & 0.12 & 0.081 & 1.7 & -25.7 \\
\hline 113 & 689B & 1B3011 & $2 \mathrm{H} 02$ & 7.66 & 0.10 & 0.016 & 6.9 & -25.4 \\
\hline 113 & 689B & 1B3012 & $2 \mathrm{H} 02$ & 8.23 & 0.19 & 0.039 & 5.7 & -25.6 \\
\hline 113 & $689 \mathrm{~B}$ & 1B3013 & $2 \mathrm{H} 03$ & 8.48 & 0.06 & 0.014 & 4.7 & -27.2 \\
\hline 113 & $689 \mathrm{~B}$ & 1B30 & $2 \mathrm{H} 03$ & 8.79 & 0.13 & 0.025 & 6.0 & -25.6 \\
\hline 113 & 689B & $1 \mathrm{~B} 3 \mathrm{C}$ & $2 \mathrm{H} 03$ & 8.79 & 0.05 & 0.082 & 0.7 & -26.7 \\
\hline 113 & 689B & 1B3016 & $2 \mathrm{HC}$ & 9. & & 0.0 & 0.4 & \\
\hline 113 & 689B & 1B3018 & $2 \mathrm{H} 04$ & 9.98 & 0.03 & & 0.5 & \\
\hline 113 & $689 \mathrm{~B}$ & $1 B 3019$ & $2 \mathrm{H} 04$ & 10.27 & 0.02 & 0.011 & 1.6 & -26.2 \\
\hline 113 & 689B & 1B3020 & $2 \mathrm{H} 04$ & 10.29 & 0.07 & 0.023 & 3.6 & -25.3 \\
\hline 113 & 689B & 1B3021 & $2 \mathrm{H} 04$ & 10.42 & 0.10 & 0.033 & 3.5 & -24.7 \\
\hline 113 & 689B & 1B3022 & $2 \mathrm{H} 04$ & 10.79 & 0.04 & 0.034 & 1.3 & -24.1 \\
\hline 113 & 689B & 1B3023 & $2 \mathrm{H} 04$ & 11.21 & 0.07 & 0.043 & 2.0 & -23.2 \\
\hline 113 & 689B & 1B3024 & $2 \mathrm{H} 05$ & 11.94 & 0.04 & 0.031 & 1.5 & -20.9 \\
\hline 113 & 689B & 1B3025 & $2 \mathrm{HOS}$ & 12.63 & 0.05 & 0.02 & 2.6 & -19.7 \\
\hline 113 & 689B & 1B3027 & $2 \mathrm{H} 06$ & 13.43 & 0.05 & 0.010 & 6.3 & -21.7 \\
\hline 113 & 689B & 1B3029 & $3 \mathrm{HOI}$ & 15.12 & 0.05 & 0.024 & 2.2 & -21.7 \\
\hline 113 & $689 \mathrm{~B}$ & $1 \mathrm{~B} 30$ & $3 \mathrm{H} 01$ & 15.65 & 0.06 & 0.009 & 7.8 & -2 \\
\hline 113 & 689B & IB3031 & $3 \mathrm{H} 01$ & 16.1 & 0.03 & 0.02 & 1.5 & \\
\hline 113 & $689 \mathrm{~B}$ & 1B3032 & $3 \mathrm{HO2}$ & 16.52 & 0.03 & 0.01 & 2.6 & \\
\hline 113 & 689B & 1B3033 & $3 \mathrm{HO} 2$ & 16.69 & 0.05 & 0.0 & 4.7 & \\
\hline 113 & 689B & 1B3034 & $3 \mathrm{HO2}$ & 17.29 & 0.05 & 0.012 & 4.4 & \\
\hline 113 & 689B & $1 B 3035$ & $3 \mathrm{HO2}$ & 17.54 & 0.06 & 0.085 & 0.9 & -26.7 \\
\hline 113 & 689B & 1B3036 & $3 \mathrm{H} 05$ & 20.96 & 0.06 & 0.010 & 7.3 & -23.2 \\
\hline 113 & 689B & 1B3037 & $3 \mathrm{H} 05$ & 21.52 & 0.04 & 0.026 & 1.8 & -26.3 \\
\hline 113 & 689B & 1B3038 & $3 \mathrm{H} 05$ & 22.04 & 0.02 & 0.015 & 1.6 & -27.2 \\
\hline 113 & 689B & 1B3039 & $3 \mathrm{H} 06$ & 22.80 & 0.06 & 0.007 & 10.0 & -28.5 \\
\hline 113 & 689B & 1B3040 & 3H06 & 23.30 & 0.04 & 0.006 & 8.3 & -24 \\
\hline 113 & $689 \mathrm{~B}$ & 1B3041 & $4 \mathrm{H} 01$ & 24.51 & 0.00 & 0.008 & 0.6 & -2 \\
\hline 113 & $689 \mathrm{~B}$ & 1B3042 & $4 \mathrm{H} 01$ & 25.58 & 0.04 & 0.008 & 6.2 & -21.6 \\
\hline 113 & $689 \mathrm{~B}$ & IB3043 & $4 \mathrm{H} 02$ & 25.99 & 0.04 & 0.007 & 6.3 & -21.1 \\
\hline 113 & $689 \mathrm{~B}$ & IB3044 & 4H02 & 27.07 & 0.04 & 0.022 & 2.3 & -2 \\
\hline 113 & 689B & 1B3045 & $4 \mathrm{H} 03$ & 27.49 & 0.04 & 0.009 & 4.8 & -24.2 \\
\hline 113 & 689B & 1B3047 & $4 \mathrm{H} 03$ & 28.57 & 0.04 & 0.010 & 4.3 & -24.7 \\
\hline 113 & 689B & 1B3048 & $4 \mathrm{H} 04$ & 29.00 & 0.06 & 0.009 & 6.8 & -27.9 \\
\hline 113 & 689B & 1B3050 & $4 \mathrm{H} 04$ & 30.07 & 0.02 & 0.004 & 5.8 & -23.3 \\
\hline 113 & 689B & 1B3051 & $4 \mathrm{H} 0 \mathrm{~S}$ & 30.50 & 0.05 & 0.014 & 3.8 & -27.8 \\
\hline 113 & 689B & 1B3052 & $4 \mathrm{H} 05$ & 31.11 & 0.07 & 0.039 & 2.1 & -24.4 \\
\hline 113 & 689B & 1B3053 & $4 \mathrm{H} 05$ & 31.57 & 0.16 & 0.382 & 0.5 & -25.1 \\
\hline 113 & $689 \mathrm{~B}$ & 1B3054 & $4 \mathrm{H} 06$ & 32.33 & 0.08 & 0.012 & 7.7 & -24.7 \\
\hline 113 & 689B & 1B3055 & 4H06 & 33.07 & 0.12 & 0.067 & 2.0 & -24.7 \\
\hline 113 & $689 \mathrm{~B}$ & 183056 & $5 \mathrm{HOI}$ & 34.00 & 0.12 & 0.013 & 10.4 & -2 \\
\hline 113 & $689 \mathrm{~B}$ & 1B3057 & $5 \mathrm{H} 01$ & 34.59 & 0.18 & 0.014 & 15.0 & -2 \\
\hline 113 & $689 \mathrm{~B}$ & 1B3058 & SH01 & 34.96 & 0.10 & 0.013 & 9.5 & -2 \\
\hline 113 & 689B & 1B3059 & SH03 & 37,00 & 0.11 & 0.017 & 7.6 & -24.2 \\
\hline 113 & 689B & $1 B 3060$ & SH03 & 37.59 & 0.13 & 0.040 & 3.9 & -24.0 \\
\hline 113 & 689B & 1B3061 & $5 \mathrm{H} 03$ & 37.96 & 0.05 & 0.021 & 2.6 & -24.8 \\
\hline 113 & 689B & 1B3062 & $5 \mathrm{H} 05$ & 40.00 & 0.08 & 0.025 & 3.2 & -24.6 \\
\hline 113 & 689B & $1 B 3063$ & $5 \mathrm{H} 05$ & 40.59 & 0.09 & 0.026 & 4.0 & -24.8 \\
\hline 113 & 689B & 1B3064 & $5 \mathrm{H} 05$ & 40.96 & 0.10 & 0.020 & 6.0 & -24.9 \\
\hline 113 & 689B & 1B3065 & $6 \mathrm{HO1}$ & 44.16 & 0.01 & 0.002 & 5.8 & -27.0 \\
\hline 113 & 689B & 1B3066 & $6 \mathrm{H} 01$ & 44.51 & 0.07 & 0.023 & 3.4 & -27.3 \\
\hline 113 & 689B & 1B30 & $6 \mathrm{H} 03$ & 46.52 & 0.11 & 0.010 & 13.9 & -27.4 \\
\hline 113 & 689B & 1B3068 & $6 \mathrm{H} 03$ & 47.16 & 0.06 & 0.040 & 1.7 & -27.1 \\
\hline 113 & 689B & 1B3069 & $6 \mathrm{H} 03$ & 47.51 & 0.03 & 0.007 & 4.3 & \\
\hline 113 & 689B & 1B3070 & $6 \mathrm{H} 05$ & 49.52 & 0.14 & 0.081 & 2.0 & -27.8 \\
\hline 113 & 689B & 1B3071 & $6 \mathrm{HOS}$ & 50.16 & 0.06 & 0.020 & 3.9 & -2 \\
\hline 113 & 689B & 1B3072 & $6 \mathrm{H} 05$ & 50.52 & 0.05 & 0.021 & 2.6 & -28.1 \\
\hline 113 & 689B & 1B3073 & $7 \mathrm{H} 01$ & 53.10 & 0.06 & 0.017 & 4.2 & -23.4 \\
\hline 113 & $689 \mathrm{~B}$ & 183074 & $7 \mathrm{H} 01$ & 53.72 & 0.13 & 0.035 & 4.2 & -24.7 \\
\hline 113 & 689B & 1B3075 & $7 \mathrm{HO} 1$ & 54.08 & 0.12 & 0.024 & 5.8 & -25.6 \\
\hline 113 & 689B & 1B3076 & $7 \mathrm{H} 03$ & 56.10 & 0.04 & 0.029 & 1.5 & -27.7 \\
\hline 113 & 689B & 1B3077 & $7 \mathrm{H} 03$ & 56.72 & 0.04 & 0.013 & 3.6 & -26.0 \\
\hline 113 & 689B & IB3078 & 7H03 & 57.08 & 0.03 & 0.026 & 1.4 & -25.9 \\
\hline 113 & 689B & 1B3079 & $7 \mathrm{HOS}$ & 59.10 & 0.01 & 0.029 & 0.1 & -27.0 \\
\hline 113 & 689B & 1B3080 & $7 \mathrm{H} 05$ & 59.72 & 0.06 & 0.060 & 1.3 & -29.1 \\
\hline 113 & 689B & 1B3081 & $7 \mathrm{HOS}$ & 60.08 & 0.06 & 0.016 & 4.1 & -28.9 \\
\hline 113 & 689B & 1B3082 & $8 \mathrm{HOI}$ & 62.70 & 0.04 & 0.029 & 1.6 & -28.2 \\
\hline 113 & 689B & 1B3083 & $8 \mathrm{H} 01$ & 63.28 & 0.01 & 0.021 & 0.6 & -26.8 \\
\hline 113 & 689B & 1B3084 & $8 \mathrm{H} 01$ & 63.66 & 0.05 & 0.031 & 1.8 & -25.9 \\
\hline 113 & 689B & 1B3085 & $8 \mathrm{H} 03$ & 65.71 & 0.06 & 0.029 & 2.5 & -28.1 \\
\hline 113 & 689B & 1B3086 & $8 \mathrm{H} 03$ & 66.29 & 0.04 & 0.030 & 1.6 & -27.1 \\
\hline 113 & 689B & 1B3087 & $8 \mathrm{H} 03$ & 66.66 & 0.06 & 0.046 & 1.5 & -27.3 \\
\hline 113 & 689B & 1B3088 & $8 \mathrm{H} 05$ & 68.71 & 0.05 & 0.022 & 2.5 & -28.4 \\
\hline 113 & 689B & 1B3089 & $8 \mathrm{H} 05$ & 69.29 & 0.00 & 0.021 & 0.3 & -28.1 \\
\hline 113 & 689B & IB3091 & $9 \mathrm{H} 01$ & 72.92 & 0.13 & 0.059 & 2.5 & -28.5 \\
\hline
\end{tabular}

Table 1 (continued).

\begin{tabular}{|c|c|c|c|c|c|c|c|c|c|}
\hline Leg & Hole & Mun\# & Core & Depth & $\% \mathrm{C}$ & $\% \mathrm{~N}$ & $\mathrm{C} / \mathrm{N}$ & $\delta^{13} \mathrm{C}$ & $\delta^{15} \mathrm{~N}$ \\
\hline 113 & 689B & 1B3093 & $9 \mathrm{H} 03$ & 75.92 & 0.15 & 0.026 & 6.7 & -28.1 & 4.9 \\
\hline 113 & $689 \mathrm{~B}$ & $1 B 3094$ & $9 \mathrm{H} 03$ & 76.25 & 0.07 & 0.035 & & & 4.2 \\
\hline 113 & $689 \mathrm{~B}$ & 1B3095 & $9 \mathrm{H} 05$ & 78.92 & 0.08 & 0.083 & 1.2 & -28.9 & 2.8 \\
\hline 113 & 6898 & $1 B 3096$ & $9 \mathrm{HOS}$ & 79.25 & 0.08 & 0.075 & 1.3 & -28.9 & 2.5 \\
\hline 113 & 689B & 1B3097 & $10 \mathrm{HOI}$ & 82.50 & 0.03 & 0.031 & 1.2 & -28.7 & 2.9 \\
\hline 113 & 689B & 1B3098 & $10 \mathrm{H} 01$ & 82.86 & 0.11 & 0.018 & 7.6 & -29.6 & 2.8 \\
\hline 113 & $689 \mathrm{~B}$ & 1B3099 & $10 \mathrm{HOS}$ & 88. & 0.18 & 0.085 & 2.5 & & 2.6 \\
\hline 113 & $689 \mathrm{~B}$ & $1 \mathrm{~B} 3100$ & $10 \mathrm{H} 05$ & 88. & 0.01 & 0.019 & 0.8 & & 3.0 \\
\hline 113 & $689 \mathrm{~B}$ & 1B3101 & $11 \mathrm{H} 01$ & 92. & 0.07 & & 1.2 & & 3.9 \\
\hline 113 & 689B & 1B31 & $11 \mathrm{H}$ & & & & & & 5.2 \\
\hline 113 & 689 & & & & & & & & 3.4 \\
\hline 113 & & & & & & & & & 4.5 \\
\hline 113 & & & & & & & 5.1 & & 4.4 \\
\hline 113 & 689B & & $12 \mathrm{HO1}$ & 102.21 & 0.07 & & 2.8 & -29.3 & 4.4 \\
\hline 113 & $689 \mathrm{~B}$ & 1B3107 & $12 \mathrm{H} 05$ & 107.88 & 0.08 & 0.023 & 4.2 & -28.6 & 4.5 \\
\hline 113 & 689B & 1B3109 & $13 \mathrm{HO1}$ & 111.48 & 0.07 & 0.060 & 1. & -28.6 & 4.2 \\
\hline 113 & 689B & 1B3110 & $13 \mathrm{H} 01$ & 111.81 & 0.57 & 0.2 & 3. & & 2.7 \\
\hline 113 & $689 \mathrm{~B}$ & $1 \mathrm{~B} 3111$ & $13 \mathrm{H}$ & 117. & 0.11 & 0.0 & 1.4 & & 3.9 \\
\hline 113 & 689B & 1B3112 & $13 \mathrm{H} 05$ & 117. & 0.08 & 0.0 & 1.3 & & 4.7 \\
\hline 113 & 689B & 1B3113 & $14 \mathrm{H} 01$ & 121. & 0.11 & 0.0 & 6. & & 5.6 \\
\hline 113 & 689B & 1B3114 & $14 \mathrm{H} 01$ & 121 & 0.1 & 0.2 & 0. & & 4.9 \\
\hline 113 & $689 \mathrm{~B}$ & 1B3115 & $14 \mathrm{H}$ & 127 & 0.1 & & 6. & & 4.3 \\
\hline 113 & $689 \mathrm{~B}$ & $1 B 3116$ & $14 \mathrm{H}$ & 127 & 0. & & & & 5.1 \\
\hline 113 & 689B & & & & & & & & 5.2 \\
\hline 113 & 689B & & & & & & 6. & & 5.4 \\
\hline 113 & 689B & & 103 & 33.67 & 0.09 & 0.062 & 1.7 & -2 & 5.2 \\
\hline 113 & $689 \mathrm{~B}$ & & $15 \mathrm{HO}$ & 134.09 & 0.06 & 0.044 & 1.6 & -27.6 & 5.5 \\
\hline 113 & 689B & 1B3121 & $15 \mathrm{H} 05$ & 136.67 & 0.15 & 0.020 & 8.9 & -2 & 6.0 \\
\hline 113 & $689 \mathrm{~B}$ & $1 \mathrm{~B} 3122$ & $15 \mathrm{H} 05$ & 137. & 0.11 & 0.0 & 4.2 & & 5.2 \\
\hline 113 & 689B & $1 \mathrm{~B} 3123$ & $16 \mathrm{H} 01$ & 140. & 0.12 & 0.1 & 0.8 & & 5.7 \\
\hline 113 & $689 \mathrm{~B}$ & $1 \mathrm{~B} 312$ & $16 \mathrm{H}$ & & 0.14 & & 7.5 & & 4.3 \\
\hline 113 & 689B & 1B3125 & $16 \mathrm{H}$ & 143 & 0.1 & 0.0 & & & 4.9 \\
\hline 113 & 689B & $1 \mathrm{~B} 3126$ & $16 \mathrm{H}$ & 143 & 0.1 & & & & 3.3 \\
\hline 113 & 689B & 1B31 & $16 \mathrm{H}$ & 146 & 0. & & & & 3.4 \\
\hline 113 & 689B & 1B31 & $17 \mathrm{H}$ & & & & & & 4.8 \\
\hline 113 & 689B & 1B3 & $17 \mathrm{H}$ & 15 & & & & & 2.9 \\
\hline 113 & 689B & 1B3 & $17 \mathrm{H}$ & & & & 1.8 & & 3.1 \\
\hline 113 & $689 \mathrm{~B}$ & IB3 & H05 & 155.86 & 0.01 & 0.04 & 0.3 & -2 & 2.9 \\
\hline 113 & 689B & IB3133 & $17 \mathrm{H} 05$ & 156.29 & 0.07 & 0.111 & 0.8 & -27.9 & 5.5 \\
\hline 113 & $689 \mathrm{~B}$ & IB3134 & $18 \mathrm{H} 01$ & 159.56 & 0.15 & 0.029 & 6.2 & -26.8 & 5.9 \\
\hline 113 & 689B & 1B3135 & $18 \mathrm{H} 01$ & & 0.04 & 0.0 & 0.7 & & 5.8 \\
\hline 113 & 689B & 1B3136 & $18 \mathrm{H} 03$ & 162.5 & 0.2 & 0.2 & 0.9 & -2 & 4.1 \\
\hline 113 & $689 \mathrm{~B}$ & 1B3137 & $18 \mathrm{H} 03$ & 162.9 & 0.0 & 0.01 & 1.3 & -2 & 3.8 \\
\hline 113 & 689B & 1B3138 & $18 \mathrm{H} 05$ & 165.5 & 0.2 & 0.0 & 4.3 & & 3.7 \\
\hline 113 & 689B & 1B3139 & $18 \mathrm{H} 05$ & 165.9 & 0.1 & 0.1 & 1.4 & -2 & 4.0 \\
\hline 113 & 689B & IB3141 & $19 \mathrm{H} 01$ & 169. & 0.6 & & 0.9 & & 3.2 \\
\hline 113 & $689 \mathrm{~B}$ & 1B31 & $19 \mathrm{H}$ & 172. & & & 4 & & 4.8 \\
\hline 113 & 689B & 1B3 & $19 \mathrm{H}$ & & & & 2.2 & & 5.7 \\
\hline 113 & 689B & IB3 & $19 \mathrm{H} 05$ & 175.27 & 0.03 & 0.0 & 0.9 & -2 & 3.6 \\
\hline 113 & 689B & IB3145 & $19 \mathrm{H} 05$ & 175.70 & 0.06 & 0.082 & 0.8 & -25.8 & 3.2 \\
\hline 113 & 689B & IB3146 & $20 \mathrm{H} 01$ & 178.84 & 0.02 & 0.034 & 0.6 & -25.1 & 3.7 \\
\hline 113 & $689 \mathrm{~B}$ & 1B3148 & $20 \mathrm{H} 03$ & 181.8 & 0.22 & 0.0 & 2.6 & -26.1 & 4.3 \\
\hline 113 & 689B & IB3149 & $20 \mathrm{H} 03$ & 182.30 & 0.06 & 0.02 & 3.7 & -2 & 5.4 \\
\hline 113 & 689B & IB3150 & $21 \mathrm{H} 01$ & 188.54 & 0.02 & 0.06 & 5.1 & -26 & 4.6 \\
\hline 113 & 689B & IB3151 & $21 \mathrm{H} 01$ & 189.00 & 0.08 & 0.057 & 1.6 & -2 & 4.4 \\
\hline 113 & 689B & 1B3152 & $21 \mathrm{H} 03$ & 191.54 & 0.04 & 0.010 & 5.4 & & 5.7 \\
\hline 113 & 689B & & & & 0.0 & & 3.7 & & 6.2 \\
\hline 113 & 689 & & & & 0.1 & & 1.5 & & 6.1 \\
\hline 113 & 689B & & $22 \mathrm{X}$ & 201.2 & 0.0 & & 1.4 & & 7.5 \\
\hline 113 & 689B & 1B3157 & $22 \times 05$ & 204.22 & 0.13 & 0.024 & 6.1 & -2 & 7.6 \\
\hline 113 & 689B & 1B3158 & $22 \times 05$ & 204.70 & 0.13 & 0.035 & 4.3 & -26 & 6.4 \\
\hline 113 & $689 \mathrm{~B}$ & 1B3159 & $23 \times 01$ & 207.98 & 0.18 & 0.042 & 4.7 & -26.7 & 7.1 \\
\hline 113 & 689B & $1 B 3160$ & $23 \times 03$ & 210.98 & 0.19 & 0.055 & 4.2 & -26.4 & 6.4 \\
\hline 113 & 689B & 1B3161 & $23 \times 03$ & 211.39 & 0.09 & 0.073 & 1.4 & -26.1 & 5.3 \\
\hline 113 & $689 \mathrm{~B}$ & 1B3162 & $24 \times 01$ & 217.65 & 0.05 & 0.014 & 4.4 & -26 & 6.0 \\
\hline 113 & 689B & 1B3163 & $26 \times 01$ & 237.21 & 0.03 & 0.018 & 1.7 & -26 & 7.8 \\
\hline 113 & $689 \mathrm{C}$ & 1C 3164 & $1 \mathrm{H} 01$ & 0.25 & 0.01 & & 2.3 & -27.3 & 6.7 \\
\hline 113 & $689 \mathrm{C}$ & & $1 \mathrm{H} 01$ & 0.50 & 0.05 & & 4.5 & & 3.9 \\
\hline 113 & & & & & & & 1.5 & & 3.1 \\
\hline 113 & $689 \mathrm{C}$ & & & 1.00 & 0.0 & & 9.3 & & 3.8 \\
\hline 113 & $689 \mathrm{C}$ & & $1 \mathrm{HC}$ & 1.25 & 0.05 & 0.01 & 4.7 & & 1.7 \\
\hline 113 & $689 \mathrm{C}$ & C3171 & $1 \mathrm{H}$ & 2.00 & 0.02 & 0.0 & 7.8 & -24 & 2.4 \\
\hline 113 & $689 \mathrm{C}$ & C 3172 & & 2.25 & 0.04 & 0.0 & 5.9 & -22 & 2.9 \\
\hline 113 & $689 \mathrm{C}$ & $1 \mathrm{C} 3173$ & & 2.50 & 0.04 & & 1.5 & & 3.5 \\
\hline 113 & $689 \mathrm{C}$ & $1 \mathrm{C} 3174$ & $1 \mathrm{H} 02$ & 2.75 & 0.04 & 0.005 & 9.5 & & 2.0 \\
\hline 113 & $689 \mathrm{C}$ & $1 \mathrm{C} 3176$ & $1 \mathrm{H} 03$ & 3.25 & 0.03 & 0.003 & 10.5 & -21.5 & 2.1 \\
\hline 113 & $689 \mathrm{C}$ & $1 \mathrm{C} 3177$ & $1 \mathrm{H} 03$ & 3.50 & 0.03 & 0.003 & 12.2 & -20.4 & 2.3 \\
\hline 113 & $689 \mathrm{C}$ & $1 \mathrm{C} 3178$ & IH03 & 3.75 & 0.04 & 0.010 & 4.9 & -22.4 & 2.2 \\
\hline 113 & $689 \mathrm{C}$ & $1 \mathrm{C} 3179$ & & 4.00 & 0.04 & & 5.2 & -21.7 & 0.8 \\
\hline 113 & $689 \mathrm{C}$ & & & 4.25 & 0.05 & 0.011 & 5.0 & & 1.5 \\
\hline 113 & & & & 4.75 & 0.05 & & 4.1 & & 2.9 \\
\hline 11 & & & & & 0.05 & & 5.8 & & 3.8 \\
\hline 113 & $689 \mathrm{C}$ & $1 C 3184$ & $1 \mathrm{H} 04$ & 5.25 & 0.04 & 0.014 & 3.6 & -23.0 & 3.1 \\
\hline
\end{tabular}


Table 1 (continued).

\begin{tabular}{|c|c|c|c|c|c|c|c|c|}
\hline Leg & Hole & Mun\# & Core & Depth & $\% \mathrm{C}$ & $\% \mathrm{~N}$ & $\mathrm{C} / \mathrm{N}$ & $\delta^{13} \mathrm{C}$ \\
\hline 113 & $689 \mathrm{C}$ & $1 C 3185$ & $1 \mathrm{H} 04$ & 5.50 & 0.04 & 0.014 & 3.4 & -22.6 \\
\hline 113 & $689 \mathrm{C}$ & $1 \mathrm{C} 3186$ & $1 \mathrm{H} 04$ & 5.75 & 0.03 & 0.009 & 4.3 & -22.0 \\
\hline 113 & $689 \mathrm{C}$ & $1 \mathrm{C} 3188$ & $1 \mathrm{H} 05$ & 6.25 & 0.04 & 0.010 & 5.0 & -22.1 \\
\hline 113 & $689 \mathrm{C}$ & IC3189 & IHOS & 6.50 & 0.03 & 0.008 & 4.4 & -23.2 \\
\hline 113 & $689 \mathrm{C}$ & $1 \mathrm{C} 3190$ & $1 \mathrm{H} 05$ & 6.75 & 0.04 & 0.011 & 4.2 & -24.0 \\
\hline 113 & $689 \mathrm{C}$ & $1 C 3191$ & $1 \mathrm{H} 05$ & 7.00 & 0.04 & 0.027 & 1.7 & -24.2 \\
\hline 113 & $689 \mathrm{C}$ & $1 C 3192$ & $1 \mathrm{H} 05$ & 7.25 & 0.04 & 0.024 & 2.0 & -23.9 \\
\hline 113 & $689 \mathrm{C}$ & $1 C 3194$ & $1 \mathrm{H} 06$ & 7.75 & 0.06 & 0.013 & 5.1 & -24.2 \\
\hline 113 & $689 \mathrm{C}$ & $1 C 3195$ & $1 \mathrm{H} 06$ & 8.25 & 0.05 & 0.011 & 5.8 & -23.9 \\
\hline 113 & $689 \mathrm{C}$ & $1 \mathrm{C} 3$ & $1 \mathrm{H} 06$ & 8.50 & 0.07 & 0.022 & 3.7 & -25.3 \\
\hline 113 & $689 \mathrm{C}$ & $1 C 3197$ & $1 \mathrm{H} 06$ & 8.75 & 0.05 & 0.011 & 4.8 & -2 \\
\hline 113 & $689 \mathrm{C}$ & $1 C 3199$ & $1 \mathrm{H} 07$ & 9.25 & 0.04 & & 5.8 & \\
\hline 113 & $689 \mathrm{C}$ & $1 C 3200$ & $1 \mathrm{H} 07$ & 9.50 & 0.05 & 0.0 & 4.7 & \\
\hline 113 & $689 \mathrm{C}$ & IC3201 & $1 \mathrm{H} 07$ & 9.75 & 0.05 & 0.012 & 4.8 & \\
\hline 113 & $689 \mathrm{C}$ & $1 \mathrm{C} 3202$ & $2 \mathrm{HO1}$ & 9.87 & 0.05 & 0.020 & 2.6 & -23.2 \\
\hline 113 & $689 \mathrm{C}$ & $1 \mathrm{C} 3203$ & $3 \mathrm{H} 01$ & 18.25 & 0.02 & 0.022 & 1.1 & -23.2 \\
\hline 113 & $689 \mathrm{C}$ & $1 C 3204$ & $3 \mathrm{HOI}$ & 18.54 & 0.06 & 0.011 & 6.5 & -22.8 \\
\hline 113 & $689 \mathrm{C}$ & $1 C 3205$ & $3 \mathrm{HOI}$ & 18.82 & 0.00 & 0.023 & 0.1 & -21.6 \\
\hline 113 & $689 \mathrm{C}$ & $1 C 3206$ & $3 \mathrm{H} 01$ & 19.02 & 0.07 & 0.043 & 2.0 & -22.5 \\
\hline 113 & $689 \mathrm{C}$ & $1 C 3207$ & $3 \mathrm{H} 01$ & 19.31 & 0.04 & 0.014 & 3.3 & -22.4 \\
\hline 113 & $689 \mathrm{C}$ & $1 C 3208$ & $3 \mathrm{H} 02$ & 19.75 & 0.03 & 0.011 & 3.8 & -20.1 \\
\hline 113 & $689 \mathrm{C}$ & $1 C 3209$ & $3 \mathrm{HO2}$ & 20.04 & 0.04 & 0.011 & 3.8 & -20.7 \\
\hline 113 & $689 \mathrm{C}$ & $1 \mathrm{C} 32$ & $3 \mathrm{HO2}$ & 20.32 & 0.04 & 0.007 & 6.5 & -19.4 \\
\hline 113 & $689 \mathrm{C}$ & $1 \mathrm{C} 3212$ & $3 \mathrm{H} 02$ & 20.81 & 0.04 & 0.013 & 3.4 & -21.1 \\
\hline 113 & $689 \mathrm{C}$ & $1 C 3213$ & $3 \mathrm{H} 03$ & 21.25 & 0.04 & 0.012 & 3.5 & -2 \\
\hline 113 & $689 \mathrm{C}$ & $1 \mathrm{C} 3214$ & $3 \mathrm{H} 03$ & 21.54 & 0.03 & 0.023 & 1.7 & \\
\hline 113 & $689 \mathrm{C}$ & $1 \mathrm{C} 3215$ & $3 \mathrm{H} 03$ & 21.82 & 0.07 & 0.021 & 3.7 & \\
\hline 113 & $689 \mathrm{C}$ & $1 C 3216$ & $3 \mathrm{H} 03$ & 22.02 & 0.05 & 0.017 & 3.3 & -25.2 \\
\hline 113 & $689 \mathrm{C}$ & $1 C 3217$ & $3 \mathrm{HO}$ & 22.31 & 0.03 & 0.009 & 4.2 & -25.0 \\
\hline 113 & $689 \mathrm{C}$ & $1 C 3218$ & $3 \mathrm{H} 04$ & 22.75 & 0.04 & 0.025 & 1.9 & -25.4 \\
\hline 113 & $689 \mathrm{C}$ & $1 C 3219$ & $3 \mathrm{H} 04$ & 23.04 & 0.00 & 0.011 & 0.3 & -25.7 \\
\hline 113 & $689 \mathrm{C}$ & $1 \mathrm{C} 3221$ & $3 \mathrm{H} 04$ & 23.52 & 0.00 & 0.010 & 0.1 & -24.4 \\
\hline 113 & $689 \mathrm{C}$ & $1 C 3222$ & $3 \mathrm{H} 04$ & 23.81 & 0.00 & 0.018 & 0.1 & -24.1 \\
\hline 113 & $689 \mathrm{C}$ & $1 C 3223$ & $3 \mathrm{HOS}$ & 24.25 & 0.00 & 0.013 & 0.2 & -26.2 \\
\hline 113 & $689 \mathrm{C}$ & $1 C 3224$ & $3 \mathrm{H} 05$ & 24.54 & 0.03 & 0.023 & 1.8 & -24.3 \\
\hline 113 & $689 \mathrm{C}$ & $1 C 3225$ & $3 \mathrm{H} 05$ & 24.82 & 0.04 & 0.014 & 3.7 & -24.8 \\
\hline 113 & $689 \mathrm{C}$ & $1 C 3226$ & $3 \mathrm{H} 05$ & 25.02 & 0.01 & 0.011 & 0.6 & -25.7 \\
\hline 113 & $689 \mathrm{C}$ & $1 C 3227$ & $3 \mathrm{H} 05$ & 25.31 & 0.04 & 0.022 & 1.9 & 4.9 \\
\hline 113 & $689 \mathrm{C}$ & $1 C 3228$ & $3 \mathrm{H} 06$ & 25.75 & 0.03 & 0.018 & 1.9 & 4.9 \\
\hline 113 & $689 \mathrm{C}$ & $1 C 3229$ & $3 \mathrm{H} 06$ & 26.04 & 0.04 & 0.010 & 4.9 & -24.7 \\
\hline 113 & $689 \mathrm{C}$ & $1 C 3230$ & $3 \mathrm{H} 06$ & 26.32 & 0.04 & 0.011 & 4.1 & -23.4 \\
\hline 113 & $689 \mathrm{C}$ & $1 C 3231$ & $3 \mathrm{H} 06$ & 26.52 & 0.04 & 0.012 & 3.9 & -24.5 \\
\hline 113 & $689 \mathrm{C}$ & $1 \mathrm{C} 3232$ & $3 \mathrm{H} 06$ & 26.81 & 0.04 & 0.011 & 4.7 & -25.1 \\
\hline 113 & $689 \mathrm{C}$ & $1 C 3233$ & $3 \mathrm{H} 07$ & 27.25 & 0.03 & 0.011 & 3.0 & -23.7 \\
\hline 113 & $689 \mathrm{C}$ & $1 C 3234$ & $3 \mathrm{H} 07$ & 27.54 & 0.03 & 0.019 & 2.1 & -23.2 \\
\hline 113 & $689 \mathrm{C}$ & $1 C 3235$ & $3 \mathrm{H} 07$ & 27.78 & 0.02 & 0.014 & 1.8 & -23.4 \\
\hline 113 & 689D & 1D3236 & $1 \mathrm{HOI}$ & 18.61 & 0.01 & 0.004 & 0.8 & -23.6 \\
\hline 113 & 689D & 1D3237 & $1 \mathrm{HO}$ & 18.86 & 0.01 & 0.002 & 1.1 & -23.7 \\
\hline 113 & 689D & 1D3238 & $1 \mathrm{HO}$ & 19.11 & 0.04 & 0.004 & 11.7 & -23.7 \\
\hline 113 & 689D & 1D3239 & $1 \mathrm{HO}$ & 19.31 & 0.01 & 0.003 & 5.2 & \\
\hline 113 & 689D & ID 3240 & $1 \mathrm{H} 02$ & 20.11 & 0.04 & 0.004 & 9.7 & 2.4 \\
\hline 113 & 689D & 1D3241 & $1 \mathrm{H} 02$ & 20.36 & 0.01 & 0.001 & 10.0 & -23.1 \\
\hline 113 & 689D & ID 3242 & $1 \mathrm{H} 02$ & 20.61 & 0.06 & 0.010 & 6.5 & -24.1 \\
\hline 113 & 689D & $1 \mathrm{D} 3243$ & $1 \mathrm{HO2}$ & 20.81 & 0.07 & 0.047 & 1.7 & -23.9 \\
\hline 113 & 689D & 1D3244 & $1 \mathrm{H} 03$ & 21.35 & 0.06 & 0.007 & 5.7 & -23.9 \\
\hline 113 & 689D & 1D3246 & $1 \mathrm{H} 03$ & 21.86 & 0.06 & 0.006 & 9.7 & -24.5 \\
\hline 113 & 689D & 1D3247 & $1 \mathrm{H} 03$ & 22.11 & 0.05 & 0.005 & 10.2 & -24.7 \\
\hline 113 & 689D & 1D3248 & $1 \mathrm{H} 03$ & 22.31 & 0.02 & 0.002 & 11.1 & -25.1 \\
\hline 113 & 689D & $1 D 3249$ & $1 \mathrm{H} 04$ & 22.85 & 0.00 & 0.001 & 2.2 & -24.7 \\
\hline 113 & 689D & 1D3250 & $1 \mathrm{H} 04$ & 23.11 & 0.06 & 0.029 & 2.2 & -23.6 \\
\hline 113 & 689D & 1D3251 & $1 \mathrm{H} 04$ & 23.36 & 0.11 & 0.029 & 4.5 & -23.4 \\
\hline 113 & 689D & 1D3252 & $1 \mathrm{H} 04$ & 23.61 & 0.00 & 0.016 & 0.1 & -23.3 \\
\hline 113 & 689D & $1 D 3253$ & $1 \mathrm{H} 04$ & 23.81 & 0.06 & 0.009 & 7.8 & -2 \\
\hline 113 & 689D & 1D3254 & $1 \mathrm{H} 05$ & 24.35 & 0.05 & 0.009 & 6.2 & \\
\hline 113 & 689D & 1D3255 & $1 \mathrm{H} 05$ & 24.61 & 0.11 & 0.041 & 3.2 & -23.9 \\
\hline 113 & 689D & $1 D 3256$ & $1 \mathrm{H} 05$ & 24.86 & 0.05 & 0.011 & 5.3 & -23.7 \\
\hline 113 & 689D & 1D3257 & $1 \mathrm{H} 05$ & 25.11 & 0.03 & 0.003 & 10.2 & -23.4 \\
\hline 113 & 689D & 1D3258 & $1 \mathrm{H} 05$ & 25.31 & 0.00 & 0.005 & 0.7 & -22.1 \\
\hline 113 & 689D & 1D3259 & $1 \mathrm{H} 06$ & 25.85 & 0.06 & 0.024 & 3.0 & -22.4 \\
\hline 113 & 689D & $1 \mathrm{D} 3260$ & $1 \mathrm{H} 06$ & 26.36 & 0.05 & 0.037 & 1.5 & -24.5 \\
\hline 113 & 689D & 1D 3262 & $1 \mathrm{H} 06$ & 26.81 & 0.09 & 0.031 & 3.6 & -23.8 \\
\hline 113 & 689D & 1D3263 & $1 \mathrm{H} 07$ & 27.35 & 0.10 & 0.015 & 7.4 & -23.4 \\
\hline 113 & 689D & ID3264 & $2 \mathrm{HO}$ & 27.85 & 0.05 & 0.007 & 7.2 & -23.6 \\
\hline 113 & 689D & 1D3265 & $2 \mathrm{HOI}$ & 28.10 & 0.05 & 0.006 & 9.8 & \\
\hline 113 & 689D & 1D3267 & $2 \mathrm{HOI}$ & 28.57 & 0.05 & 0.009 & 5.6 & -28.3 \\
\hline 113 & 689D & 1D3269 & $2 \mathrm{H} 02$ & 29.35 & 0.04 & 0.010 & 4.4 & -28.4 \\
\hline 113 & 689D & 1D3270 & $2 \mathrm{H} 02$ & 29.60 & 0.05 & 0.014 & 3.9 & -28.0 \\
\hline 113 & 689D & 1D3271 & $2 \mathrm{H} 02$ & 29.83 & 0.06 & 0.056 & 1.1 & -27.7 \\
\hline 113 & 689D & 1D3272 & $2 \mathrm{H} 02$ & 30.07 & 0.03 & 0.010 & 3.8 & -28.7 \\
\hline 113 & 689D & $1 \mathrm{D} 3273$ & $2 \mathrm{HO2}$ & 30.32 & 0.06 & 0.039 & 1.7 & -28.2 \\
\hline 113 & 689D & 1D3274 & $2 \mathrm{H} 03$ & 30.85 & 0.03 & 0.041 & 0.9 & -27.6 \\
\hline 113 & 689D & 1D3275 & $2 \mathrm{H} 03$ & 31.10 & 0.08 & 0.253 & 0.4 & -27.4 \\
\hline 113 & 689D & 1D3276 & $2 \mathrm{H} 03$ & 31.33 & 0.01 & 0.009 & 0.7 & -25.7 \\
\hline
\end{tabular}

Table 1 (continued).

\begin{tabular}{|c|c|c|c|c|c|c|c|c|c|}
\hline Leg & Hole & Mun\# & Core & Depth & $\% \mathrm{C}$ & $\% \mathrm{~N}$ & $\mathrm{C} / \mathrm{N}$ & $\delta^{13} \mathrm{C}$ & $\delta^{15}$ \\
\hline 113 & 689D & 1D3277 & $2 \mathrm{H} 03$ & 31.57 & 0.02 & 0.011 & 2.0 & -26.5 & \\
\hline 113 & 689D & 1D3278 & $2 \mathrm{H} 03$ & & & & 0.6 & -25.7 & \\
\hline 113 & 689D & 1D3279 & $2 \mathrm{H} 04$ & 32.35 & 0.00 & 0.006 & 0.4 & -25.1 & \\
\hline 113 & 689D & ID 3280 & $2 \mathrm{H} 04$ & 32.60 & 0.06 & 0.012 & 5.2 & -25.6 & \\
\hline 113 & 689D & 1D3281 & $2 \mathrm{H} 04$ & 32.83 & 0.01 & & 1.1 & -25.7 & \\
\hline 113 & 689D & 1D 3282 & $2 \mathrm{H} 04$ & 33.07 & 0.20 & 0.049 & 4.7 & -25.8 & \\
\hline 113 & $689 \mathrm{D}$ & ID 3283 & $2 \mathrm{H} 04$ & 33.32 & 0.01 & 0.003 & 5.0 & & \\
\hline 113 & 689D & ID 3284 & $2 \mathrm{H} 05$ & 33.85 & 0.01 & 0.085 & 0.1 & & \\
\hline 113 & 689D & ID3285 & $2 \mathrm{H} 05$ & 34.10 & 0.00 & 0.002 & 2.2 & -24.7 & \\
\hline 113 & 689D & 1D3286 & $2 \mathrm{H} 05$ & 34.33 & 0.13 & 0.084 & 1.8 & -24.7 & \\
\hline 113 & 689D & ID 3287 & $2 \mathrm{H} 05$ & 34.57 & 0.02 & 0.006 & 4.3 & -25.1 & \\
\hline 113 & 689D & ID 3288 & $2 \mathrm{H} 05$ & 34.82 & 0.01 & 0.021 & 0.6 & -24.8 & \\
\hline 113 & 689D & & $2 \mathrm{H} 06$ & 35.35 & 0.01 & 0.075 & 0.1 & -25.1 & \\
\hline 113 & 689D & ID 3290 & $2 \mathrm{H} 06$ & 35.60 & 0.12 & 0.050 & 2.8 & & \\
\hline 113 & $689 \mathrm{D}$ & ID3291 & $2 \mathrm{H} 06$ & & 0.01 & 0.004 & 4.1 & & \\
\hline 113 & 689D & 1D32 & $2 \mathrm{H} 06$ & & & 0.090 & 1.2 & -25.9 & \\
\hline 113 & 689D & ID 3293 & $2 \mathrm{H} 06$ & 36.32 & 0.01 & 0.066 & 0.0 & -26.0 & \\
\hline 113 & 689D & 1D 3294 & $2 \mathrm{H} 07$ & & 0.01 & & 0.1 & & \\
\hline 113 & 689D & 1D32S & $2 \mathrm{H} 07$ & & & & 0.3 & & \\
\hline 113 & $689 \mathrm{D}$ & ID 3296 & $2 \mathrm{H} 07$ & 37.33 & 0.09 & 0.074 & 1.4 & & \\
\hline 113 & 689D & 1D 3297 & $3 \mathrm{H} 01$ & 37.50 & 0.00 & & 0.2 & & \\
\hline 113 & 689D & 1D329 & $3 \mathrm{H} 01$ & 37. & 0.00 & & 1.0 & & \\
\hline 113 & 689D & 1D3299 & $3 \mathrm{H} 01$ & 38.06 & 0.11 & 0.056 & 2.3 & -26.1 & \\
\hline 113 & $689 \mathrm{D}$ & ID 3300 & $3 \mathrm{H} 01$ & & 0.01 & 0.030 & 0.2 & -25.7 & \\
\hline 113 & 689D & 330 & $3 \mathrm{HO} 2$ & & 0.01 & 0.059 & 0.2 & & \\
\hline 113 & 689D & 330 & $3 \mathrm{H} 02$ & 39.25 & 0.20 & 0.092 & 2.5 & -27.0 & \\
\hline 113 & 689D & iD & $3 \mathrm{HO}$ & 39.56 & 0.01 & 0.038 & 0.1 & -26.0 & \\
\hline 113 & 689D & 33 & $3 \mathrm{H} 02$ & 39.81 & 0.00 & 0.004 & 0.5 & & \\
\hline 113 & $689 \mathrm{D}$ & iI & $3 \mathrm{H} 02$ & 40. & 0.01 & 0.008 & 1.2 & -24 & \\
\hline 113 & 689D & ID 33 & $3 \mathrm{HO}$ & 40.50 & 0.01 & 0.00 & 0.5 & & \\
\hline 113 & 689D & ID3308 & $3 \mathrm{H} 03$ & 40.75 & 0.01 & 0.007 & 0.6 & & \\
\hline 113 & $689 \mathrm{D}$ & & $\mathrm{H} 03$ & 41.06 & 0.01 & 0.008 & 0.2 & -25 & \\
\hline 113 & 689D & ID 33 & $3 \mathrm{H}$ & 41. & 1 & & 3.7 & -2 & \\
\hline 113 & 689D & ID3311 & $\mathrm{H} 03$ & 41.55 & .01 & 5 & 0.6 & & \\
\hline $\begin{array}{l}113 \\
113\end{array}$ & $689 \mathrm{D}$ & & & 42.00 & & & 2.3 & & \\
\hline 113 & 689D & ID33 & $3 \mathrm{H} 0$ & 42.25 & 0.06 & 0.0 & 1.7 & & \\
\hline 113 & 689D & ID3314 & $3 \mathrm{H} 04$ & 42.56 & 0.09 & 0.060 & 1.8 & -27.4 & \\
\hline 113 & 689D & & $3 \mathrm{H} 04$ & & & 0.029 & 0.2 & & \\
\hline 113 & 689D & ID331 & 3H04 & 43.05 & 0.07 & 0.024 & 3.2 & -2 & \\
\hline 113 & 689D & II & $3 \mathrm{H} 05$ & 43.50 & 0.07 & 0.050 & 4.6 & -30.2 & \\
\hline 113 & 689D & & & & & & & & \\
\hline 113 & 689D & ID 3319 & $3 \mathrm{H} 05$ & 44.06 & 0.08 & 0.096 & 1.0 & -2 & \\
\hline 113 & 689D & & $3 \mathrm{HO}$ & 44. & 0. & 0.083 & 0.1 & -27.4 & \\
\hline 113 & 689D & iI & 3H06 & 45.00 & 0. & & 1.4 & $-3 c$ & \\
\hline 113 & 689D & 11 & $\mathrm{HO}$ & 45.2 & 0.08 & 0.223 & 0.4 & -2 & \\
\hline 113 & $99 \mathrm{D}$ & & $3 \mathrm{HC}$ & 46. & 0.06 & 0.251 & 0.3 & -28 & \\
\hline 113 & $689 \mathrm{D}$ & II & $4 \mathrm{H} 01$ & 48.24 & 0.12 & 0.262 & 0.5 & -30 & \\
\hline 113 & 689D & il & $4 \mathrm{HO}$ & 48.7 & 0.08 & 0.052 & 1.7 & -2 & \\
\hline 113 & & & & & & & 0.6 & & \\
\hline 113 & 689D & & $4 \mathrm{HO}$ & 49.27 & 0.08 & 0.1 & 0.7 & -26 & \\
\hline 113 & 689D & & $4 \mathrm{H}$ & 49. & & 0.110 & 0.6 & -2 & \\
\hline 113 & & & & & & & 0.6 & & \\
\hline 113 & 689D & iI & $4 \mathrm{H}$ & 50 & 0.1 & 0.24 & 0.5 & -2 & \\
\hline 113 & 689 & & & & & 0.2 & 0. & -2 & \\
\hline 113 & & & & & & & 0.2 & & \\
\hline 113 & 68 & & $4 \mathrm{H}$ & .24 & 0. & & 0.2 & -2 & \\
\hline 113 & 689 & & & & & 0.1 & 1.7 & -2 & \\
\hline 113 & & & & & & & & & \\
\hline 113 & 68 & & $4 \mathrm{H}$ & & & & 2. & -2 & \\
\hline 113 & & & & & & & 2. & & \\
\hline 113 & & & & & & & & & \\
\hline 113 & & & & & & & 0. & -2 & \\
\hline 113 & & & & & & & & & \\
\hline 113 & & & & & & & & & \\
\hline 113 & & & & & & & & & \\
\hline $\begin{array}{l}113 \\
\text { nas }\end{array}$ & & & & & & & & & \\
\hline 113 & & & & & & & & & \\
\hline 113 & & & & & & & & & \\
\hline 113 & & & & & & & & & \\
\hline $\begin{array}{l}113 \\
113\end{array}$ & & & & & & & & & \\
\hline 113 & & & & & & & & & \\
\hline 113 & & & & & & & & & \\
\hline 113 & & & & & & & & & \\
\hline & & & & & & & & & \\
\hline 113 & & & & & & & & & \\
\hline 113 & & & & & & & & & \\
\hline & & & & & & & & & \\
\hline 113 & & & & & & & & & \\
\hline 113 & & & & & & & & & \\
\hline & & & & & & & & & \\
\hline $\begin{array}{l}113 \\
113\end{array}$ & & 2 & & & & & 1. & & \\
\hline 113 & $690 \mathrm{~A}$ & & $1 \mathrm{H} 03$ & & & & 0.3 & -24.0 & \\
\hline 113 & & 243374 & & & & 0.288 & 0.2 & -25.1 & 6 . \\
\hline
\end{tabular}


Table 1 (continued).

\begin{tabular}{|c|c|c|c|c|c|c|c|c|c|c|c|c|c|c|}
\hline Leg & Hole & Mun\# & Core & Depth & $\% \mathrm{C}$ & $\% \mathrm{~N}$ & $\mathrm{C} / \mathrm{N}$ & $\delta^{13} \mathrm{C}$ & $\delta^{15} \mathrm{~N}$ & Leg & Hole & Mun\# & Core & Depth \\
\hline 113 & $690 \mathrm{~A}$ & $2 A 3376$ & $1 \mathrm{H} 04$ & 4.93 & 0.04 & 0.021 & 2.4 & -25.4 & 4.5 & 113 & $693 \mathrm{~A}$ & $3 \mathrm{~A} 3471$ & 2R01 & 3.10 \\
\hline 113 & $690 \mathrm{~A}$ & $2 A 3377$ & $1 \mathrm{H} 04$ & 5.18 & 0.06 & 0.034 & 2.2 & -26.2 & 5.8 & 113 & $693 \mathrm{~A}$ & $3 \mathrm{~A} 3472$ & 2R01 & 3.38 \\
\hline 113 & $690 \mathrm{~A}$ & $2 A 3378$ & 1H04 & 5.52 & 0.05 & 0.044 & 1.2 & -24.5 & 7.4 & 113 & $693 \mathrm{~A}$ & $3 A 3473$ & 2R01 & 3.82 \\
\hline 113 & $690 \mathrm{~A}$ & $2 A 3379$ & $1 \mathrm{H} 04$ & 5.87 & 0.04 & 0.030 & 1.6 & -23.2 & 5.4 & 113 & $693 \mathrm{~A}$ & $3 \mathrm{~A} 3474$ & 2R02 & 4.30 \\
\hline 113 & $690 \mathrm{~A}$ & $2 \mathrm{~A} 3380$ & IHOS & 6.15 & 0.05 & 0.050 & 1.2 & -22.9 & 5.0 & 113 & $693 \mathrm{~A}$ & $3 A 3475$ & 2R02 & 4.60 \\
\hline 113 & $690 \mathrm{~A}$ & $2 A 3381$ & $1 \mathrm{HOS}$ & 6.43 & 0.06 & 0.022 & 3.4 & -21.7 & 3.8 & 113 & $693 \mathrm{~A}$ & $3 \mathrm{~A} 3476$ & 2R02 & 4.90 \\
\hline 113 & $690 \mathrm{~A}$ & $2 A 3382$ & $1 \mathrm{H} 0 \mathrm{~S}$ & 6.68 & 0.17 & 0.026 & 7.5 & -23.1 & 5.0 & 113 & $693 \mathrm{~A}$ & $3 \mathrm{~A} 3477$ & 2R02 & 5.32 \\
\hline 113 & $690 \mathrm{~A}$ & $2 A 3383$ & $1 \mathrm{H} 0 \mathrm{~S}$ & 7.02 & 0.12 & 0.011 & 9.7 & -20.6 & 4.5 & 113 & $693 \mathrm{~A}$ & $3 \mathrm{~A} 3478$ & 2R03 & 5.82 \\
\hline 113 & $690 \mathrm{~A}$ & 2A3384 & $1 \mathrm{HOS}$ & 7.37 & 0.19 & 0.320 & 0.7 & -21.5 & 4.4 & 113 & $693 \mathrm{~A}$ & $3 \mathrm{~A} 3479$ & 2R03 & 6.10 \\
\hline 113 & $690 \mathrm{~A}$ & $2 \mathrm{~A} 3385$ & $1 \mathrm{H} 06$ & 7.65 & 0.04 & 0.036 & 1.4 & -22.8 & 4.7 & 113 & $693 \mathrm{~A}$ & $3 \mathrm{~A} 3480$ & $2 \mathrm{R} 03$ & 6.40 \\
\hline 113 & $690 \mathrm{~A}$ & $2 A 3386$ & $1 \mathrm{H} 06$ & 7.93 & 0.06 & 0.495 & 0.1 & -22.3 & 5.0 & 113 & $693 \mathrm{~A}$ & $3 \mathrm{~A} 3481$ & $2 \mathrm{R} 03$ & 6.82 \\
\hline 113 & $690 \mathrm{~A}$ & $2 \mathrm{~A} 3388$ & $1 \mathrm{H} 06$ & 8.52 & 0.05 & 0.010 & 4.7 & -22.4 & 4.8 & 113 & $693 \mathrm{~A}$ & $3 \mathrm{~A} 3482$ & 2R04 & 7.32 \\
\hline 113 & $690 \mathrm{~A}$ & $2 \mathrm{~A} 3390$ & $1 \mathrm{H} 07$ & 9.43 & 0.06 & 0.561 & 0.1 & -22.3 & 3.8 & 113 & $693 \mathrm{~A}$ & $3 \mathrm{~A} 3483$ & 2R04 & 7.60 \\
\hline 113 & $690 \mathrm{~A}$ & $2 A 3391$ & $1 \mathrm{H} 07$ & 9.68 & 0.06 & 0.029 & 2.5 & -22.4 & 3.6 & 113 & 693A & $3 \mathrm{~A} 3484$ & $2 R 04$ & 7.90 \\
\hline 113 & $690 \mathrm{~B}$ & 2B3395 & $2 \mathrm{H} 01$ & 2.53 & 0.06 & 0.038 & 2.0 & -24.0 & 6.5 & 113 & $693 \mathrm{~A}$ & $3 \mathrm{~A} 3485$ & 2R04 & 8.32 \\
\hline 113 & $690 \mathrm{~B}$ & 2B3397 & $2 \mathrm{H} 02$ & 4.03 & 0.06 & 0.028 & 2.4 & -25.7 & 5.0 & 113 & $693 \mathrm{~A}$ & $3 \mathrm{~A} 3486$ & 2R05 & 8.82 \\
\hline 113 & $690 \mathrm{~B}$ & 2B3399 & $2 \mathrm{H} 03$ & 5.53 & 0.02 & 0.010 & 2.8 & -21.5 & 4.1 & 113 & $693 \mathrm{~A}$ & $3 \mathrm{~A} 3487$ & 2R0S & 9.10 \\
\hline 113 & $690 \mathrm{~B}$ & $2 \mathrm{~B} 3400$ & $2 \mathrm{H} 03$ & 6.12 & 0.04 & 0.185 & 0.2 & -21.9 & 3.4 & 113 & $693 \mathrm{~A}$ & $3 \mathrm{~A} 3488$ & 2R0S & 9.40 \\
\hline 113 & $690 \mathrm{~B}$ & $2 \mathrm{~B} 3402$ & $2 \mathrm{H} 04$ & 7.62 & 0.09 & 0.061 & 1.6 & -23.8 & 4.7 & 113 & $693 \mathrm{~A}$ & $3 \mathrm{~A} 3489$ & 2R0S & 9.82 \\
\hline 113 & 690B & 2B3403 & $2 \mathrm{HOS}$ & 8.53 & 0.06 & 0.213 & 0.3 & -23.6 & 4.1 & 113 & $693 \mathrm{~A}$ & $3 \mathrm{~A} 3490$ & 2R06 & 10.32 \\
\hline 113 & 690B & 2B3404 & $2 \mathrm{H} 05$ & 9.12 & 0.05 & 0.075 & 0.8 & -23.8 & 3.9 & 113 & $693 \mathrm{~A}$ & $3 \mathrm{~A} 3491$ & 2R06 & 10.60 \\
\hline 113 & $690 \mathrm{~B}$ & $2 B 3405$ & $2 \mathrm{H} 06$ & 10.03 & 0.06 & 0.040 & 2.1 & -24.3 & 3.5 & 113 & $693 \mathrm{~A}$ & $3 A 3492$ & 2R06 & 10.90 \\
\hline 113 & $690 \mathrm{~B}$ & 2B3406 & $2 \mathrm{H} 06$ & 10.62 & 0.05 & 0.033 & 2.0 & -24.6 & 5.8 & 113 & $693 \mathrm{~A}$ & $3 \mathrm{~A} 3493$ & 2R06 & 11.32 \\
\hline 113 & 690B & 2B3407 & $2 \mathrm{H} 07$ & 11.53 & 0.04 & 0.004 & 10.6 & -24.7 & 6.3 & 113 & $693 \mathrm{~A}$ & $3 A 3494$ & 2R07 & 11.82 \\
\hline 113 & $690 \mathrm{~B}$ & 2B3408 & $3 \mathrm{H} 01$ & 12.13 & 0.05 & 0.202 & 0.3 & -24.7 & 5.6 & 113 & $693 \mathrm{~A}$ & $3 \mathrm{~A} 3495$ & 3R01 & 12.52 \\
\hline 113 & 690B & 2B3409 & $3 \mathrm{H} 01$ & 12.72 & 0.46 & 0.159 & 0.3 & -24.0 & 2.0 & 113 & $693 \mathrm{~A}$ & $3 A 3496$ & 3R01 & 12.80 \\
\hline 113 & $690 \mathrm{~B}$ & 2B3410 & $3 \mathrm{H} 02$ & 13.63 & 0.23 & 0.112 & 2.4 & -26.0 & 3.9 & 113 & $693 \mathrm{~A}$ & $3 A 3497$ & $3 \mathrm{R} 01$ & 13.10 \\
\hline 113 & 690B & 2B3411 & $3 \mathrm{H} 02$ & 14.22 & 0.03 & 0.049 & 0.7 & -25.1 & 3.7 & 113 & $693 \mathrm{~A}$ & 3A3498 & 3R01 & 13.53 \\
\hline 113 & $690 \mathrm{~B}$ & $2 B 3412$ & $3 \mathrm{H} 03$ & 15.13 & 0.05 & 0.291 & 0.2 & -23.8 & 4.5 & 113 & $693 \mathrm{~A}$ & $3 \mathrm{~A} 3500$ & $3 R 02$ & 14.30 \\
\hline 113 & $690 \mathrm{~B}$ & 2B3413 & $3 \mathrm{H} 03$ & 15.72 & 0.13 & 0.025 & 6.1 & -24.1 & 5.4 & 113 & $693 \mathrm{~A}$ & 3A3501 & $3 \mathrm{R} 02$ & 14.60 \\
\hline 113 & $690 \mathrm{~B}$ & 2B3414 & $3 \mathrm{H} 04$ & 16.63 & 0.48 & 0.239 & 2.3 & -24.2 & 5.3 & 113 & $693 \mathrm{~A}$ & $3 \mathrm{~A} 3502$ & $3 \mathrm{R} 02$ & 14.91 \\
\hline 113 & $690 \mathrm{~B}$ & 2B3415 & $3 \mathrm{H} 04$ & 17.22 & 0.06 & 0.257 & 0.3 & -23.9 & 5.1 & 113 & $693 \mathrm{~A}$ & $3 \mathrm{~A} 3503$ & $4 \mathrm{R} 01$ & 22.22 \\
\hline 113 & $690 \mathrm{~B}$ & 2B3416 & $3 \mathrm{H} 05$ & 18.13 & 0.06 & 0.291 & 0.3 & -27.0 & 5.0 & 113 & $693 \mathrm{~A}$ & $3 \mathrm{~A} 3504$ & $4 \mathrm{R} 01$ & 22.50 \\
\hline 113 & $690 \mathrm{~B}$ & $2 B 3417$ & $3 \mathrm{H} 05$ & 18.72 & 0.05 & 0.289 & 0.2 & -28.5 & 6.8 & 113 & $693 \mathrm{~A}$ & $3 \mathrm{~A} 3505$ & $4 \mathrm{R} 01$ & 22.80 \\
\hline 113 & $690 \mathrm{~B}$ & $2 B 3418$ & $3 \mathrm{H} 06$ & 19.63 & 0.05 & 0.310 & 0.2 & -29.0 & 4.9 & 113 & $693 \mathrm{~A}$ & $3 \mathrm{~A} 3506$ & $4 \mathrm{R} 01$ & 23.22 \\
\hline 113 & $690 \mathrm{~B}$ & 2B3419 & $3 \mathrm{H} 06$ & 20.22 & 0.04 & 0.193 & 0.3 & -29.3 & 4.8 & 113 & $693 \mathrm{~A}$ & $3 \mathrm{~A} 3507$ & $4 \mathrm{R} 02$ & 23.72 \\
\hline 113 & 690B & 2B3420 & $3 \mathrm{H} 07$ & 21.13 & 0.09 & 0.313 & 0.3 & -29.4 & 3.4 & 113 & $693 \mathrm{~A}$ & $3 \mathrm{~A} 3508$ & $4 \mathrm{R} 02$ & 24.00 \\
\hline 113 & 690B & 2B3421 & $4 \mathrm{H} 01$ & 21.83 & 0.05 & 0.146 & 0.4 & -23.6 & 8.7 & 113 & $693 \mathrm{~A}$ & $3 \mathrm{~A} 3509$ & $4 \mathrm{R} 02$ & 24.30 \\
\hline 113 & 690B & $2 \mathrm{~B} 3422$ & $4 \mathrm{H} 01$ & 22.42 & 0.04 & 0.027 & 1.8 & -26.0 & 5.0 & 113 & $693 \mathrm{~A}$ & 3 A 3510 & $4 \mathrm{R} 02$ & 24.72 \\
\hline 113 & $690 \mathrm{~B}$ & 2B 3423 & $4 \mathrm{H} 02$ & 23.33 & 0.04 & 0.269 & 0.2 & -28.2 & 4.5 & 113 & $693 \mathrm{~A}$ & 3 A 3512 & $4 \mathrm{R} 03$ & 25.50 \\
\hline 113 & $690 \mathrm{~B}$ & 2B3424 & $4 \mathrm{H} 02$ & 23.92 & 0.09 & 0.025 & 4.1 & -26.9 & 5.7 & 113 & $693 \mathrm{~A}$ & $3 \mathrm{~A} 3513$ & $4 \mathrm{R} 03$ & 25.80 \\
\hline 113 & $690 \mathrm{~B}$ & 2B3425 & $4 \mathrm{H} 03$ & 24.83 & 0.04 & 0.008 & 5.8 & -26.4 & 5.4 & 113 & $693 \mathrm{~A}$ & $3 \mathrm{~A} 3514$ & $4 \mathrm{R} 04$ & 26.72 \\
\hline 113 & $690 \mathrm{~B}$ & 2B3426 & $4 \mathrm{H} 03$ & 25.42 & 0.09 & 0.257 & 0.4 & -26.6 & 5.1 & 113 & $693 \mathrm{~A}$ & $3 \mathrm{~A} 3515$ & $4 \mathrm{R} 04$ & 27.00 \\
\hline 113 & $690 \mathrm{~B}$ & 2B3427 & $4 \mathrm{H} 04$ & 26.33 & 0.06 & 0.180 & 0.4 & -27.8 & 5.3 & 113 & $693 \mathrm{~A}$ & $3 \mathrm{~A} 3516$ & $4 \mathrm{R} 04$ & 27.30 \\
\hline 113 & $690 \mathrm{~B}$ & 2B3429 & $4 \mathrm{H} 05$ & 27.83 & 0.10 & 0.303 & 0.4 & -27.2 & 3.0 & 113 & $693 \mathrm{~A}$ & $3 \mathrm{~A} 3517$ & 4R04 & 27.72 \\
\hline 113 & $690 \mathrm{~B}$ & 2B3430 & $4 \mathrm{H} 05$ & 28.42 & 0.08 & 0.249 & 0.4 & -25.5 & 7.0 & 113 & $693 \mathrm{~A}$ & $3 \mathrm{~A} 3518$ & $4 \mathrm{R} 05$ & 28.22 \\
\hline 113 & $690 \mathrm{~B}$ & 2B3431 & $4 \mathrm{H} 06$ & 29.33 & 0.08 & 0.052 & 1.8 & -27.8 & 3.7 & 113 & $693 \mathrm{~A}$ & $3 \mathrm{~A} 3520$ & 5R01 & 31.73 \\
\hline 113 & 690B & 2B3432 & $4 \mathrm{H} 06$ & 29.92 & 0.14 & 0.113 & 1.5 & -27.3 & 3.6 & 113 & $693 \mathrm{~A}$ & $3 \mathrm{~A} 3521$ & $5 R 01$ & 32.00 \\
\hline 113 & $690 \mathrm{~B}$ & 2B3433 & $5 \mathrm{HOI}$ & 31.53 & 0.05 & 0.040 & 1.4 & -26.8 & 3.9 & 113 & $693 \mathrm{~A}$ & $3 \mathrm{~A} 3522$ & SR01 & 32.29 \\
\hline 113 & 690B & 2B3434 & $5 \mathrm{H} 01$ & 32.12 & 0.02 & 0.244 & 0.1 & -27.3 & 3.4 & 113 & $693 \mathrm{~A}$ & 3 A 3523 & SR01 & 32.71 \\
\hline 113 & $690 \mathrm{~B}$ & 2B3435 & SH02 & 33.03 & 0.06 & 0.271 & 0.2 & -28.1 & 5.0 & 113 & $693 \mathrm{~A}$ & $3 \mathrm{~A} 3524$ & 5R02 & 33.23 \\
\hline 113 & $690 \mathrm{~B}$ & 2B3437 & $5 \mathrm{H} 03$ & 34.53 & 0.06 & 0.183 & 0.4 & -27.9 & 4.3 & 113 & $693 \mathrm{~A}$ & $3 \mathrm{~A} 3525$ & 5R02 & 33.50 \\
\hline 113 & 690B & 2B3438 & $5 \mathrm{H} 03$ & 35.12 & 0.07 & 0.309 & 0.2 & -28.7 & 5.4 & 113 & $693 \mathrm{~A}$ & $3 \mathrm{~A} 3526$ & SR02 & 33.79 \\
\hline 113 & $690 \mathrm{~B}$ & 2B3439 & $5 \mathrm{H} 04$ & 36.03 & 0.06 & 0.184 & 0.4 & -29.4 & 3.5 & 113 & $693 \mathrm{~A}$ & $3 A 3527$ & SR02 & 34.21 \\
\hline 113 & 690B & 2B3440 & $5 \mathrm{H} 04$ & 36.62 & 0.10 & 0.306 & 0.4 & -26.4 & 1.7 & 113 & $693 \mathrm{~A}$ & $3 \mathrm{~A} 3528$ & $5 \mathrm{R} 03$ & 34.73 \\
\hline 113 & $690 \mathrm{~B}$ & 2B3441 & $5 \mathrm{H} 0 \mathrm{~S}$ & 37.53 & 0.07 & 0.309 & 0.3 & -27.3 & 3.2 & 113 & $693 \mathrm{~A}$ & $3 A 3529$ & $5 R 03$ & 35.00 \\
\hline 113 & $690 \mathrm{~B}$ & 2B3442 & $5 \mathrm{H} 05$ & 38.12 & 0.03 & 0.028 & 1.4 & -29.8 & 4.6 & 113 & $693 \mathrm{~A}$ & $3 \mathrm{~A} 3530$ & SR03 & 35.29 \\
\hline 113 & $690 \mathrm{~B}$ & 2B3443 & $5 \mathrm{H} 06$ & 39.03 & 0.05 & 0.267 & 0.2 & -29.6 & 6.0 & 113 & $693 \mathrm{~A}$ & $3 \mathrm{~A} 3531$ & $5 R 03$ & 35.71 \\
\hline 113 & $690 \mathrm{~B}$ & 2B3444 & $5 \mathrm{H} 06$ & 39.62 & 0.03 & 0.263 & 0.1 & -24.4 & 2.7 & 113 & $693 \mathrm{~A}$ & 3 A3532 & SR04 & 36.23 \\
\hline 113 & $690 \mathrm{~B}$ & 2B3445 & $6 \mathrm{H} 01$ & 41.81 & 0.08 & 0.418 & 0.2 & -29.8 & 3.3 & 113 & $693 \mathrm{~A}$ & $3 \mathrm{~A} 3533$ & SR04 & 36.50 \\
\hline 113 & 690B & 2B3446 & $6 \mathrm{H} 02$ & 43.31 & 0.07 & 0.039 & 2.1 & -27.4 & 5.8 & 113 & $693 \mathrm{~A}$ & 3 A 3534 & SR04 & 36.79 \\
\hline 113 & $690 \mathrm{~B}$ & $2 B 3447$ & $6 \mathrm{H} 03$ & 44.81 & 0.12 & 0.344 & 0.4 & -28.5 & 5.1 & 113 & $693 \mathrm{~A}$ & $3 \mathrm{~A} 3535$ & SR04 & 37.21 \\
\hline 113 & $690 \mathrm{~B}$ & 2B3448 & $6 \mathrm{H} 04$ & 46.31 & 0.03 & 0.029 & 1.2 & -29.1 & 5.2 & 113 & $693 \mathrm{~A}$ & $3 \mathrm{~A} 3536$ & SROS & 37.73 \\
\hline 113 & 690B & 2B3450 & $6 \mathrm{H} 06$ & 49.31 & 0.06 & 0.281 & 0.3 & -28.2 & 5.1 & 113 & $693 \mathrm{~A}$ & $3 \mathrm{~A} 3537$ & SR0S & 38.00 \\
\hline 113 & $690 \mathrm{~B}$ & 2B3451 & $7 \mathrm{H} 01$ & 50.83 & 0.07 & 0.028 & 3.0 & -27.7 & 5.7 & 113 & $693 \mathrm{~A}$ & $3 \mathrm{~A} 3538$ & SR0S & 38.29 \\
\hline 113 & $690 \mathrm{~B}$ & $2 \mathrm{~B} 3452$ & 7H02 & 52.33 & 0.08 & 0.026 & 3.7 & -27.8 & 5.2 & 113 & $693 \mathrm{~A}$ & $3 \mathrm{~A} 3539$ & SR05 & 38.71 \\
\hline 113 & 690B & 2B3453 & $7 \mathrm{H} 03$ & 53.83 & 0.09 & 0.222 & 0.4 & -26.6 & 5.2 & 113 & $693 \mathrm{~A}$ & $3 \mathrm{~A} 3540$ & 5R06 & 39.23 \\
\hline 113 & 690B & 2B3454 & $7 \mathrm{H} 04$ & 55.33 & 0.09 & 0.222 & 0.4 & -28.4 & 5.2 & 113 & $693 \mathrm{~A}$ & $3 \mathrm{~A} 3541$ & 5R06 & 39.50 \\
\hline 113 & 690B & 2B3455 & $7 \mathrm{H} 06$ & 58.33 & 0.17 & 0.042 & 4.7 & -28.4 & 5.3 & 113 & $693 \mathrm{~A}$ & $3 \mathrm{~A} 3542$ & 5R06 & 39.79 \\
\hline 113 & $690 \mathrm{~B}$ & 2B3456 & $7 \mathrm{H} 07$ & 59.83 & 0.06 & 0.009 & 6.9 & -28.6 & 5.4 & 113 & $693 \mathrm{~A}$ & $3 A 3543$ & 6R01 & 41.43 \\
\hline 113 & $690 \mathrm{~B}$ & $2 B 3457$ & $8 \mathrm{HOI}$ & 61.12 & 0.07 & 0.021 & 3.9 & -28.7 & 5.3 & 113 & $693 \mathrm{~A}$ & 3A3544 & 6R01 & 41.70 \\
\hline 113 & $690 \mathrm{~B}$ & 2B3458 & $8 \mathrm{H} 02$ & 62.62 & 0.08 & 0.184 & 0.5 & -28.5 & 5.6 & 113 & $693 \mathrm{~A}$ & 3 A 3545 & 6R01 & 42.00 \\
\hline 113 & 690B & 2B3459 & $8 \mathrm{H} 03$ & 64.12 & 0.06 & 0.030 & 2.5 & -28.4 & 5.6 & 113 & $693 \mathrm{~A}$ & 3 A3546 & 6R01 & 42.42 \\
\hline 113 & $690 \mathrm{~B}$ & $2 B 3460$ & $8 \mathrm{H} 04$ & 65.62 & 0.05 & 0.035 & 1.6 & -28.5 & 5.4 & 113 & $693 \mathrm{~A}$ & $3 \mathrm{~A} 3547$ & 6R02 & 42.93 \\
\hline 113 & $690 \mathrm{~B}$ & 2B3461 & $8 \mathrm{HOS}$ & 67.12 & 0.08 & 0.226 & 0.4 & -28.6 & 5.3 & 113 & $693 \mathrm{~A}$ & $3 \mathrm{~A} 3548$ & $6 \mathrm{R} 02$ & 43.20 \\
\hline 113 & $690 \mathrm{~B}$ & 2B3462 & $8 \mathrm{H} 06$ & 68.62 & 0.07 & 0.034 & 2.4 & -28.8 & 5.4 & 113 & $693 \mathrm{~A}$ & $3 \mathrm{~A} 3549$ & 6R02 & 43.50 \\
\hline 113 & $693 \mathrm{~A}$ & $3 \mathrm{~A} 3464$ & IR01 & 0.60 & 0.06 & 0.029 & 2.2 & -23.5 & 5.7 & 113 & $693 \mathrm{~A}$ & $3 \mathrm{~A} 3550$ & 6R02 & 43.92 \\
\hline 113 & $693 \mathrm{~A}$ & $3 \mathrm{~A} 3465$ & $1 \mathrm{R} 01$ & 0.90 & 0.22 & 0.433 & 0.6 & -22.2 & 7.4 & 113 & $693 \mathrm{~A}$ & $3 \mathrm{~A} 3551$ & $6 \mathrm{R} 03$ & 44.43 \\
\hline 113 & $693 \mathrm{~A}$ & $3 A 3466$ & IR01 & 1.32 & 0.21 & 0.417 & 0.6 & -22.1 & 3.4 & 113 & $693 \mathrm{~A}$ & $3 \mathrm{~A} 3552$ & 6R03 & 44.70 \\
\hline 113 & $693 \mathrm{~A}$ & $3 \mathrm{~A} 3467$ & $1 \mathrm{R} 02$ & 1.82 & 0.19 & 0.322 & 0.7 & -22.3 & 6.1 & 113 & $693 \mathrm{~A}$ & $3 \mathrm{~A} 3553$ & 6R03 & 45.00 \\
\hline 113 & $693 \mathrm{~A}$ & $3 A 3468$ & $1 \mathrm{R} 02$ & 2.10 & 0.11 & 0.149 & 0.9 & -22.9 & 8.7 & 113 & $693 \mathrm{~A}$ & $3 \mathrm{~A} 3554$ & 6R03 & 45.42 \\
\hline 113 & $693 \mathrm{~A}$ & $3 \mathrm{~A} 3469$ & $1 \mathrm{R} 02$ & 2.40 & 0.12 & 0.321 & 0.4 & -22.7 & 9.1 & 113 & $693 \mathrm{~A}$ & 3 A3555 & 6R04 & 45.93 \\
\hline 113 & $693 \mathrm{~A}$ & $3 \mathrm{~A} 3470$ & 2R01 & 2.80 & 0.07 & 0.253 & 0.3 & -22.8 & 7.2 & 113 & $693 \mathrm{~A}$ & $3 \mathrm{~A} 3556$ & 6R04 & 46.20 \\
\hline
\end{tabular}


Table 1 (continued).

\begin{tabular}{|c|c|c|c|c|c|c|c|c|c|c|c|c|c|c|}
\hline Leg & Hole & Mun\# & Core & Depth & $\% \mathrm{C}$ & $\% \mathrm{~N}$ & $\mathrm{C} / \mathrm{N}$ & $\delta^{13} \mathrm{C}$ & $\delta^{15} \mathrm{~N}$ & Leg & Hole & Mun\# & Core & Depth \\
\hline 113 & $693 \mathrm{~A}$ & $3 \mathrm{~A} 3557$ & 6R04 & 46.50 & 0.08 & 0.112 & 0.8 & -23.1 & 5.0 & 113 & $693 \mathrm{~A}$ & $3 A 3652$ & 28R04 & 258.87 \\
\hline 113 & $693 \mathrm{~A}$ & 3A3558 & 6R05 & 47.43 & 0.08 & 0.157 & 0.6 & -24.0 & 4.7 & 113 & $693 \mathrm{~A}$ & $3 \mathrm{~A} 3653$ & 29R01 & 263.67 \\
\hline 113 & $693 \mathrm{~A}$ & 3 A3559 & 6R0S & 47.70 & 0.09 & 0.291 & 0.4 & -21.9 & 6.7 & 113 & $693 \mathrm{~A}$ & $3 \mathrm{~A} 3654$ & $29 \mathrm{R} 02$ & 265.17 \\
\hline 113 & $693 \mathrm{~A}$ & $3 A 3560$ & 6R0S & 48.00 & 0.06 & 0.275 & 0.3 & -23.1 & 4.0 & 113 & $693 \mathrm{~A}$ & $3 \mathrm{~A} 3655$ & $29 \mathrm{R} 03$ & 266.67 \\
\hline 113 & $693 \mathrm{~A}$ & $3 \mathrm{~A} 3561$ & 6R0S & 48.42 & 0.17 & 0.463 & 0.4 & -22.4 & 2.9 & 113 & $693 \mathrm{~A}$ & $3 \mathrm{~A} 3656$ & $29 \mathrm{R} 04$ & 268.17 \\
\hline 113 & $693 \mathrm{~A}$ & $3 \mathrm{~A} 3562$ & 6R06 & 48.93 & 0.13 & 0.276 & 0.6 & -22.2 & 3.1 & 113 & $693 \mathrm{~A}$ & $3 \mathrm{~A} 3657$ & 29R0S & 269.67 \\
\hline 113 & $693 \mathrm{~A}$ & $3 \mathrm{~A} 3563$ & 6R06 & 49.20 & 0.17 & 0.197 & 1.0 & -22.9 & 6.6 & 113 & $693 \mathrm{~A}$ & 3A3658 & 31R01 & 282.96 \\
\hline 113 & $693 \mathrm{~A}$ & 3 A 3564 & 7R01 & 51.12 & 0.09 & 0.208 & 0.5 & -22.0 & 7.3 & 113 & $693 \mathrm{~A}$ & $3 \mathrm{~A} 3659$ & $33 \mathrm{R} 01$ & 302.30 \\
\hline 113 & $693 \mathrm{~A}$ & $3 \mathrm{~A} 3565$ & 7R01 & 51.40 & 0.16 & 0.102 & 1.9 & -22.3 & 3.0 & 113 & $693 \mathrm{~A}$ & $3 \mathrm{~A} 3660$ & $34 \mathrm{R} 01$ & 311.97 \\
\hline 113 & 693A & $3 A 3566$ & 7R01 & 51.70 & 0.23 & 0.249 & 1.1 & -23.1 & 4.7 & 113 & 693A & $3 \mathrm{~A} 3661$ & 35R01 & 322.23 \\
\hline 113 & $693 \mathrm{~A}$ & $3 A 3567$ & 7R01 & 52.12 & 0.09 & 0.162 & 0.7 & -23.3 & 2.7 & 113 & 693A & $3 \mathrm{~A} 3663$ & 36R01 & 331.27 \\
\hline 113 & $693 \mathrm{~A}$ & $3 A 3568$ & 7R02 & 52.62 & 0.09 & 0.162 & 0.6 & -24.0 & 4.6 & 113 & $693 \mathrm{~A}$ & 3A3664 & $38 \mathrm{R} 01$ & 350.68 \\
\hline 113 & $693 \mathrm{~A}$ & $3 A 3569$ & 7R02 & 53.20 & 0.08 & 0.117 & 0.8 & -23.4 & 6.4 & 113 & 693A & 3A3665 & $39 \mathrm{R} 01$ & 360.33 \\
\hline 113 & $693 \mathrm{~A}$ & $3 \mathrm{~A} 3570$ & $8 R 01$ & 61.16 & 0.09 & 0.266 & 0.4 & -23.1 & 5.0 & 113 & $693 \mathrm{~A}$ & $3 A 3666$ & $39 \mathrm{R} 02$ & 361.83 \\
\hline 113 & $693 \mathrm{~A}$ & 3A3571 & $8 \mathrm{R} 02$ & 62.66 & 0.04 & 0.096 & 0.5 & -22.5 & 3.5 & 113 & $693 \mathrm{~A}$ & $3 \mathrm{~A} 3667$ & 40R01 & 369.66 \\
\hline 113 & 693A & $3 A 3572$ & $8 \mathrm{R} 03$ & 64.16 & 0.12 & 0.348 & 0.4 & -22.9 & 3.8 & 113 & 693A & 3A3668 & 40R02 & 371.16 \\
\hline 113 & 693A & $3 A 3573$ & 8R04 & 65.66 & 0.06 & 0.046 & 1.6 & -23.4 & 4.4 & 113 & $693 \mathrm{~A}$ & $3 A 3669$ & 40R03 & 372.66 \\
\hline 113 & $693 \mathrm{~A}$ & 3 A 3574 & $8 R 05$ & 67.16 & 0.05 & 0.127 & 0.5 & -22.0 & 1.0 & 113 & $693 \mathrm{~A}$ & $3 \mathrm{~A} 3670$ & 50R01 & 465.85 \\
\hline 113 & $693 \mathrm{~A}$ & 3A3575 & $8 \mathrm{R} 06$ & 68.66 & 0.08 & 0.031 & 3.0 & -23.5 & 1.3 & 113 & $693 \mathrm{~A}$ & $3 \mathrm{~A} 3$ & S0R02 & 466.40 \\
\hline 113 & $693 \mathrm{~A}$ & $3 A 3576$ & 9R01 & 70.87 & 0.08 & 0.040 & 2.0 & -22.1 & 2.9 & 113 & 693B & $3 \mathrm{~B} 3 \mathrm{C}$ & $2 \times 01$ & 234.12 \\
\hline 113 & $693 \mathrm{~A}$ & $3 \mathrm{~A} 3577$ & $9 \mathrm{R} 02$ & 72.37 & 0.01 & 0.005 & 2.2 & -22.4 & 3.8 & 113 & 693B & 3B3673 & $2 \times 01$ & 234.42 \\
\hline 113 & 693A & 3A3578 & $9 \mathrm{R} 03$ & 73.87 & 0.15 & 0.150 & 1.1 & -22.2 & 3.0 & 113 & 693B & $3 B 3674$ & $2 \times 01$ & 234.70 \\
\hline 113 & 693A & 3A3579 & 9R04 & 75.37 & 0.18 & 0.277 & 0.8 & -21.7 & 2.2 & 113 & 693B & $3 B 3675$ & $2 \times 01$ & 235.14 \\
\hline 113 & $693 \mathrm{~A}$ & $3 \mathrm{~A} 3580$ & 9R0S & 76.87 & 0.11 & 0.295 & 0.4 & -21.6 & 2.9 & 113 & $693 \mathrm{~B}$ & 3B3676 & $2 \mathrm{X} 02$ & 235.62 \\
\hline 113 & $693 \mathrm{~A}$ & $3 \mathrm{~A} 3581$ & 9R06 & 78.37 & 0.19 & 0.165 & 1.3 & -21.9 & 2.0 & 113 & $693 \mathrm{~B}$ & 3B3677 & $2 \times 02$ & 235.92 \\
\hline 113 & $693 \mathrm{~A}$ & 3A3582 & 10R01 & 80.45 & 0.05 & 0.031 & 2.0 & -23.2 & 4.2 & 113 & 693B & 3B3678 & $2 \times 02$ & 236.20 \\
\hline 113 & $693 \mathrm{~A}$ & 3 A 3583 & 10R02 & 81.98 & 0.15 & 0.147 & 1.2 & -23.2 & 4.5 & 113 & 693B & 3B3680 & $2 \times 03$ & 237.12 \\
\hline 113 & $693 \mathrm{~A}$ & 3 A 3584 & 10R03 & 83.47 & 0.10 & 0.162 & 0.7 & -23.2 & 4.4 & 113 & $693 \mathrm{~B}$ & 3B3681 & $2 \times 03$ & 237.42 \\
\hline 113 & 693A & $3 A 3586$ & 10R06 & 87.97 & 0.11 & 0.277 & 0.5 & -23.3 & 6.1 & 113 & 693B & 3B3682 & $2 \times 03$ & 237.70 \\
\hline 113 & $693 \mathrm{~A}$ & $3 A 3587$ & $11 R 01$ & 90.02 & 0.16 & 0.150 & 1.1 & -23.5 & 5.1 & 113 & 693B & 3B3683 & $2 \times 03$ & 238.08 \\
\hline 113 & $693 \mathrm{~A}$ & 3A 3588 & $11 R 02$ & 91.57 & 0.09 & 0.178 & 0.6 & -23.3 & 3.6 & 113 & 693B & 3B3684 & $2 \times 04$ & 238.62 \\
\hline 113 & $693 \mathrm{~A}$ & 3A3589 & $11 R 03$ & 93.07 & 0.10 & 0.264 & 0.4 & -23.2 & 4.2 & 113 & 693B & 3B3685 & $2 \times 04$ & 238.92 \\
\hline 113 & $693 \mathrm{~A}$ & $3 \mathrm{~A} 3590$ & $11 \mathrm{R} 04$ & 94.57 & 0.10 & 0.294 & 0.4 & -23.6 & 4.3 & 113 & 693B & 3B3687 & $6 \mathrm{H} 01$ & 268.12 \\
\hline 113 & $693 \mathrm{~A}$ & 3A3591 & $11 \mathrm{R} 05$ & 96.07 & 0.01 & 0.032 & 0.4 & -23.8 & 4.6 & 113 & $693 \mathrm{~B}$ & 3B3688 & $6 \mathrm{H} 01$ & 268.42 \\
\hline 113 & 693A & 3A3592 & $11 \mathrm{R} 05$ & 96.69 & 0.10 & 0.348 & 0.3 & -23.2 & 4.1 & 113 & 693B & 3B3689 & $6 \mathrm{H} 01$ & 268.69 \\
\hline 113 & 693A & 3A3594 & $11 R 06$ & 97.57 & 0.14 & 0.273 & 0.6 & -22.4 & 5.5 & 113 & 693B & $3 \mathrm{~B} 3690$ & $6 \mathrm{H} 01$ & 269.12 \\
\hline 113 & $693 \mathrm{~A}$ & 3A3595 & 12R01 & 99.67 & 0.02 & 0.241 & 1.1 & -23.4 & 3.6 & 113 & 693B & 3B3691 & $6 \mathrm{H} 02$ & 269.62 \\
\hline 113 & 693A & 3A3596 & $12 \mathrm{R} 02$ & 101.17 & 0.18 & 0.148 & 1.4 & -24.3 & 2.0 & 113 & 693B & 3B3692 & $6 \mathrm{H} 02$ & 269.92 \\
\hline 113 & $693 \mathrm{~A}$ & 3A3597 & $12 \mathrm{R} 03$ & 102.67 & 0.17 & 0.295 & 0.7 & -25.1 & 3.3 & 113 & 693B & 3B3693 & $6 \mathrm{H} 02$ & 270.19 \\
\hline 113 & 693A & 3A3598 & 12R04 & 104.17 & 0.19 & 0.210 & 1.1 & -25.6 & 3.1 & 113 & 693B & 3B3694 & $6 \mathrm{H} 02$ & 270.62 \\
\hline 113 & 693A & 3A3599 & 12R0S & 105.67 & 0.12 & 0.133 & 1.1 & -22.9 & 3.2 & 113 & 693B & 3B3695 & $6 \mathrm{H} 03$ & 271.12 \\
\hline 113 & 693A & $3 \mathrm{~A} 3600$ & $12 \mathrm{R} 06$ & 107.17 & 0.34 & 0.450 & 0.9 & -26.0 & 2.0 & 113 & 6938 & 3B3696 & $6 \mathrm{H} 03$ & 271.42 \\
\hline 113 & 693A & $3 \mathrm{~A} 3601$ & 12R07 & 108.15 & 0.04 & 0.031 & 1.4 & -24.0 & 2.8 & 113 & 693B & 3B3697 & $6 \mathrm{H} 03$ & 271.69 \\
\hline 113 & $693 \mathrm{~A}$ & $3 \mathrm{~A} 3602$ & 12R07 & 108.39 & 0.09 & 0.040 & 2.8 & -23.2 & 3.7 & 113 & 693B & 3B3698 & $6 \mathrm{H} 03$ & 272.12 \\
\hline 113 & $693 \mathrm{~A}$ & $3 \mathrm{~A} 3606$ & 13R03 & 112.37 & 0.23 & 0.066 & 4.1 & -22.8 & 3.8 & 113 & 693B & 3B3699 & $6 \mathrm{H} 04$ & 272.62 \\
\hline 113 & $693 \mathrm{~A}$ & $3 \mathrm{~A} 3607$ & 13R03 & 112.55 & 0.20 & 0.071 & 3.1 & -22.7 & 3.6 & 113 & 6938 & 3B3700 & $6 \mathrm{H} 04$ & 272.92 \\
\hline 113 & $693 \mathrm{~A}$ & 3A3608 & $13 R 04$ & 113.87 & 0.11 & 0.056 & 2.4 & -22.7 & 3.8 & 113 & 693B & 3B3701 & $6 \mathrm{H} 04$ & 273.19 \\
\hline 113 & $693 \mathrm{~A}$ & $3 \mathrm{~A} 3610$ & $14 \mathrm{R} 01$ & 118.97 & 0.17 & 0.057 & 3.4 & -22.2 & 3.1 & 113 & 693B & 383702 & $6 \mathrm{H} 04$ & 273.62 \\
\hline 113 & $693 \mathrm{~A}$ & $3 \mathrm{~A} 3611$ & $14 \mathrm{R} 02$ & 120.47 & 0.20 & 0.047 & 4.8 & -23.4 & 2.9 & 113 & 693B & $3 \mathrm{~B} 3703$ & $6 \mathrm{H} 05$ & 274.12 \\
\hline 113 & $693 \mathrm{~A}$ & $3 \mathrm{~A} 3612$ & $14 \mathrm{R} 03$ & 121.97 & 0.08 & 0.036 & 2.5 & -23.4 & 3.8 & 113 & 693B & 3B3704 & $6 \mathrm{H} 05$ & 274.42 \\
\hline 113 & $693 \mathrm{~A}$ & $3 A 3613$ & $14 \mathrm{R} 04$ & 123.47 & 0.14 & 0.033 & 5.1 & -22.9 & 3.8 & 113 & 693B & 3B3705 & $6 \mathrm{HOS}$ & 274.69 \\
\hline 113 & $693 \mathrm{~A}$ & $3 \mathrm{~A} 3615$ & 14R05 & 124.97 & 0.12 & 0.047 & 3.1 & -23.4 & 3.1 & 113 & 693B & 3B3706 & $6 \mathrm{H} 05$ & 275.12 \\
\hline 113 & $693 \mathrm{~A}$ & $3 \mathrm{~A} 3617$ & 15R02 & 130.07 & 0.11 & 0.041 & 3.3 & -23.6 & 4.5 & 113 & 693B & 3B3707 & $6 \mathrm{H} 06$ & 275.62 \\
\hline 113 & $693 \mathrm{~A}$ & $3 \mathrm{~A} 3618$ & 15R03 & 131.57 & 0.08 & 0.031 & 3.0 & -23.1 & 4.6 & 113 & 693B & 3B3708 & $6 \mathrm{H} 06$ & 275.92 \\
\hline 113 & $693 \mathrm{~A}$ & $3 A 3621$ & 17R02 & 149.47 & 0.07 & 0.015 & 5.7 & -22.5 & 3.2 & 113 & 693B & 3В3709 & $6 \mathrm{H} 06$ & 276.19 \\
\hline 113 & 693A & $3 A 3622$ & 17R02 & 149.69 & 0.54 & 0.099 & 6.4 & -22.4 & 4.4 & 113 & 693B & 3B3710 & $6 \mathrm{H} 06$ & 276.62 \\
\hline 113 & $693 \mathrm{~A}$ & $3 \mathrm{~A} 3623$ & $17 \mathrm{R} 03$ & 150.73 & 0.12 & 0.059 & 2.4 & -22.1 & 3.4 & 113 & 693B & 3B3711 & $7 \mathrm{HO1}$ & 278.27 \\
\hline 113 & $693 \mathrm{~A}$ & $3 \mathrm{~A} 3624$ & 18R01 & 157.67 & 0.19 & 0.053 & 4.3 & -21.8 & 3.1 & 113 & 693B & 3B3712 & $7 \mathrm{H} 02$ & 279.77 \\
\hline 113 & $693 \mathrm{~A}$ & $3 \mathrm{~A} 3625$ & 18R02 & 159.17 & 0.12 & 0.046 & 3.1 & -22.1 & 4.2 & 113 & 693B & 3B3713 & $7 \mathrm{H} 03$ & 281.27 \\
\hline 113 & $693 \mathrm{~A}$ & $3 A 3626$ & $18 \mathrm{R} 03$ & 160.67 & 0.08 & 0.021 & 4.6 & -24.8 & 4.4 & 113 & 693B & 3B3714 & $8 \times 01$ & 287.86 \\
\hline 113 & $693 \mathrm{~A}$ & $3 \mathrm{~A} 3627$ & $18 R 04$ & 162.17 & 0.22 & 0.052 & 4.9 & -24.9 & 4.8 & 113 & 693B & 3B3715 & $8 \times 03$ & 290.86 \\
\hline 113 & $693 \mathrm{~A}$ & 3 A 3628 & 19R01 & 167.37 & 0.11 & 0.043 & 3.1 & -25.7 & 4.3 & 113 & 693B & 3B3716 & $9 \times 01$ & 297.55 \\
\hline 113 & $693 \mathrm{~A}$ & 3 A3629 & 19R02 & 168.87 & 0.21 & 0.040 & 62 & -22.8 & 4.5 & 113 & $693 \mathrm{~B}$ & 3B3717 & $9 \times 02$ & 299.05 \\
\hline 113 & $693 \mathrm{~A}$ & $3 \mathrm{~A} 3630$ & $19 \mathrm{R} 03$ & 170.37 & 0.07 & 0.034 & 2.4 & -22.2 & 3.9 & 113 & $692 \mathrm{~B}$ & $3 B 3718$ & $9 \times 03$ & 300.55 \\
\hline 113 & $693 \mathrm{~A}$ & $3 \mathrm{~A} 3631$ & 19R04 & 171.87 & 0.07 & 0.032 & 2.7 & -22.6 & 4.2 & 113 & 693B & 3B3719 & $9 \times 04$ & 302.05 \\
\hline 113 & $693 \mathrm{~A}$ & $3 \mathrm{~A} 3632$ & 19R0S & 173.42 & 0.07 & 0.027 & 2.8 & -22.2 & 3.9 & 113 & 693B & $3 B 3720$ & $9 \times 05$ & 303.55 \\
\hline 113 & 693A & $3 A 3633$ & 21R01 & 186.67 & 0.12 & 0.019 & 7.5 & -22.6 & 4.7 & 113 & 693B & 3B3721 & $10 \times 01$ & 307.25 \\
\hline 113 & $693 \mathrm{~A}$ & $3 \mathrm{~A} 3634$ & $21 \mathrm{R} 02$ & 187.92 & 0.18 & 0.057 & 3.6 & -23.2 & 4.6 & 113 & $693 \mathrm{~B}$ & $3 B 3722$ & $10 \times 02$ & 308.75 \\
\hline 113 & $693 \mathrm{~A}$ & $3 \mathrm{~A} 3635$ & 22R01 & 196.37 & 0.27 & 0.068 & 4.6 & -22.8 & 4.6 & 113 & 693B & $3 B 3723$ & $10 \times 03$ & 310.25 \\
\hline 113 & $693 \mathrm{~A}$ & $3 A 3636$ & $22 \mathrm{R} 02$ & 197.87 & 0.10 & 0.039 & 3.0 & -22.3 & 3.3 & 113 & 693B & 3 B3724 & $10 \times 04$ & 311.76 \\
\hline 113 & $693 \mathrm{~A}$ & $3 \mathrm{~A} 3637$ & $22 \mathrm{R} 03$ & 199.37 & 0.25 & 0.059 & 4.9 & -22.1 & 4.7 & 113 & 693B & $3 B 3725$ & $11 \times 01$ & 316.87 \\
\hline 113 & $693 \mathrm{~A}$ & 3 A 3638 & $22 \mathrm{R} 03$ & 199.42 & 0.04 & 0.016 & 3.4 & -22.9 & 4.1 & 113 & 693B & 3B3726 & $12 \times 01$ & 326.55 \\
\hline 113 & $693 \mathrm{~A}$ & $3 \mathrm{~A} 3639$ & 25R01 & 225.37 & 0.11 & 0.047 & 2.7 & -23.1 & 4.7 & 113 & 693B & 3B3727 & $12 \times 02$ & 328.05 \\
\hline 113 & $693 \mathrm{~A}$ & $3 \mathrm{~A} 3640$ & 25R02 & 226.87 & 0.21 & 0.080 & 3.0 & -24.3 & 2.9 & 113 & 693B & $3 B 3728$ & $12 \times 03$ & 329.55 \\
\hline 113 & $693 \mathrm{~A}$ & $3 \mathrm{~A} 3641$ & $25 \mathrm{R} 03$ & 228.37 & 0.10 & 0.035 & 3.3 & -23.2 & 4.7 & 113 & 693B & 3B3729 & $12 \times 04$ & 331.03 \\
\hline 113 & $693 \mathrm{~A}$ & $3 \mathrm{~A} 3642$ & 25R04 & 229.87 & 0.08 & 0.034 & 2.8 & -23.6 & 3.6 & 113 & 693B & 3B3730 & $12 \times 05$ & 332.55 \\
\hline 113 & $693 \mathrm{~A}$ & $3 A 3643$ & $25 R 04$ & 230.48 & 0.11 & 0.040 & 3.2 & -25.1 & 3.4 & 113 & 693B & 3B3731 & $13 \times 01$ & 336.15 \\
\hline 113 & $693 \mathrm{~A}$ & 3A3644 & 26R01 & 235.07 & 0.10 & 0.034 & 3.3 & -25.8 & 4.9 & 113 & 693B & 3B3732 & $13 \times 02$ & 337.70 \\
\hline 113 & $693 \mathrm{~A}$ & $3 A 3645$ & 26R02 & 236.57 & 0.07 & 0.014 & 5.7 & -25.5 & 4.6 & 113 & 693B & 3B3733 & $14 \times 01$ & 345.86 \\
\hline 113 & $693 \mathrm{~A}$ & $3 \mathrm{~A} 3647$ & 26R04 & 239.57 & 0.09 & 0.035 & 3.0 & -25.6 & 3.8 & 113 & 693B & $3 B 3734$ & $14 \times 02$ & 347.41 \\
\hline 113 & $693 \mathrm{~A}$ & $3 \mathrm{~A} 3648$ & 26R0S & 241.07 & 0.22 & 0.049 & 5.2 & -24.8 & 3.5 & 113 & 693B & 3B3735 & $14 \times 03$ & 348.88 \\
\hline 113 & $693 \mathrm{~A}$ & $3 A 3649$ & 27R01 & 244.68 & 0.13 & 0.050 & 3.1 & -25.7 & 3.3 & 113 & $693 \mathrm{~B}$ & $3 B 3736$ & $14 \times 04$ & 350.30 \\
\hline & & & & & & & 1.8 & & 3.2 & 113 & 693B & 3B3737 & $14 \times 05$ & 351.79 \\
\hline
\end{tabular}

Table 1 (continued) 
Table 1 (continued).

Table 1 (continued).

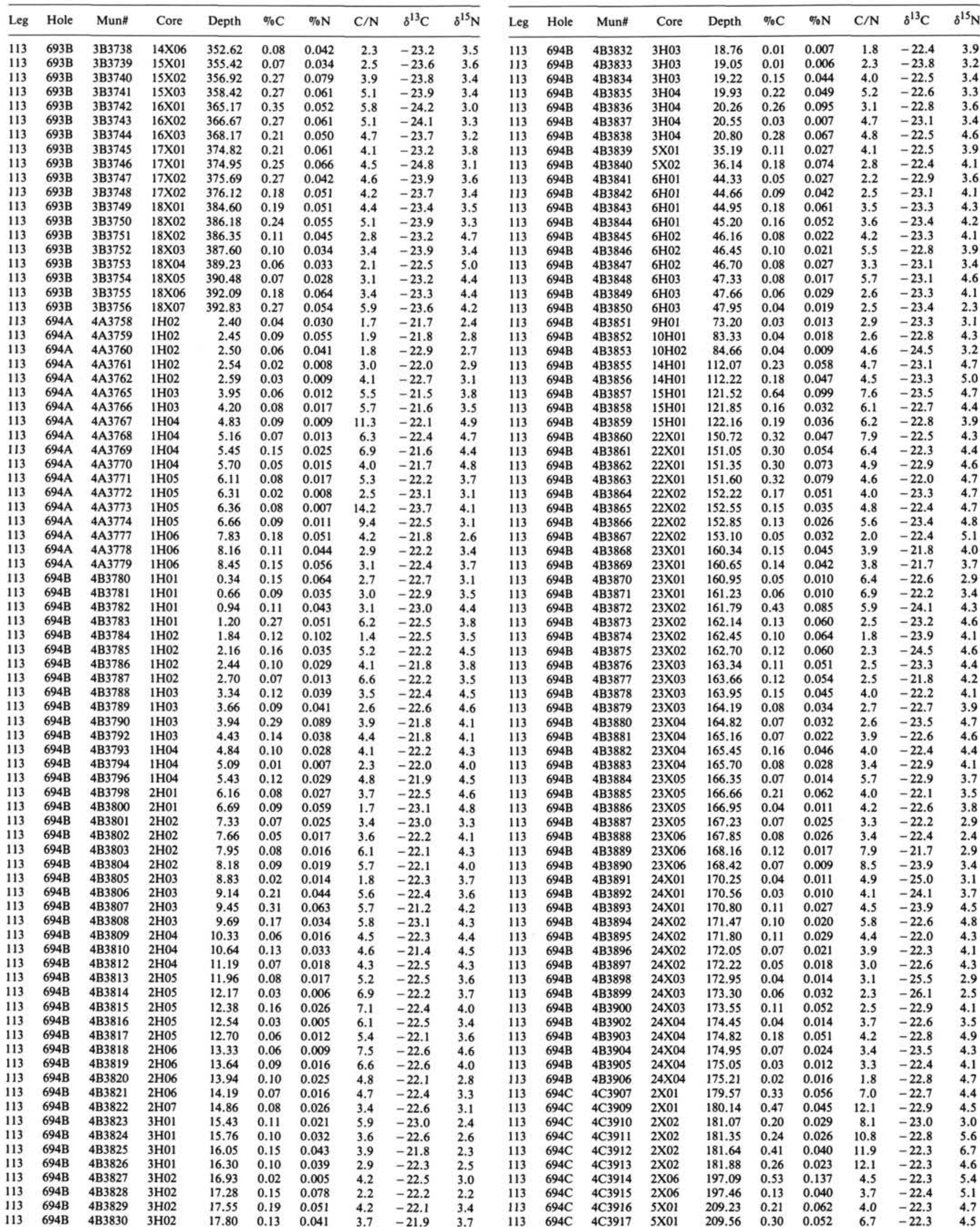


Table 1 (continued).

\begin{tabular}{|c|c|c|c|c|c|c|c|c|c|}
\hline Leg & Hole & Mun\# & Core & Depth & $\% \mathrm{C}$ & $\% \mathrm{~N}$ & $\mathrm{C} / \mathrm{N}$ & $\delta^{13} \mathrm{C}$ & $\delta^{15} \mathrm{~N}$ \\
\hline 113 & $694 \mathrm{C}$ & 4C 3918 & $5 \times 01$ & 209.85 & 0.31 & 0.078 & 4.6 & & 4.0 \\
\hline 113 & $694 \mathrm{C}$ & 4C3919 & $5 \times 01$ & & 0.08 & & 2.6 & & 3.9 \\
\hline 113 & $694 \mathrm{C}$ & $4 \mathrm{C} 39$ & & & & & 8.6 & & 3.8 \\
\hline 113 & 694 & & & & & & 5.8 & & 3.7 \\
\hline 113 & $694 \mathrm{C}$ & & $5 \times 02$ & & & & & & 4.0 \\
\hline 113 & $694 \mathrm{C}$ & $4 C 3924$ & $6 \times 01$ & & & & & & 2.7 \\
\hline 113 & $694 \mathrm{C}$ & $4 C 3925$ & $6 \times 01$ & & & & & & 3.0 \\
\hline 113 & $694 \mathrm{C}$ & 4C 3926 & $6 \times 01$ & 218. & & & & & 2.7 \\
\hline 113 & $694 \mathrm{C}$ & 4C3927 & $6 \times 01$ & 219.10 & 0.14 & 0.0 & 3.4 & & 3.1 \\
\hline 113 & $694 \mathrm{C}$ & 4C3928 & $6 \times 02$ & 219.73 & 0.25 & 0.0 & 8.7 & & 6.3 \\
\hline 113 & $694 \mathrm{C}$ & $4 C 3929$ & $6 \times 02$ & 220. & 0.18 & 0.0 & 6.0 & & 6.8 \\
\hline 113 & $694 \mathrm{C}$ & $4 C 3930$ & $6 \times 02$ & 220 . & 0.11 & 0.0 & 7.1 & & 6.1 \\
\hline 113 & $694 \mathrm{C}$ & $4 C 3932$ & $6 \times 03$ & 221. & 0.3 & 0.0 & 6.6 & & 4.5 \\
\hline 113 & $694 \mathrm{C}$ & $4 C 3933$ & $6 \times 03$ & 221. & 0.1 & 0.0 & 8. & & 4.2 \\
\hline 113 & $694 \mathrm{C}$ & & & 221 & 0.1 & 0.0 & 7.2 & & 4.3 \\
\hline 113 & $694 \mathrm{C}$ & & & & 0. & & 6.8 & & 4.5 \\
\hline 113 & $694 \mathrm{C}$ & & & & & & 12 & & 4.2 \\
\hline 113 & $694 \mathrm{C}$ & & & & & & & & 5.5 \\
\hline 113 & 694 & & & & & & & & 4.7 \\
\hline 113 & $694 \mathrm{C}$ & & $6 \mathrm{X}$ & & & & & & 4.1 \\
\hline 113 & $694 \mathrm{C}$ & $4 C 3940$ & $6 \mathrm{X}$ & 224.23 & 0.2 & & & & 3.7 \\
\hline 113 & $694 \mathrm{C}$ & $4 C 3941$ & $6 \times 05$ & 224.56 & 0.21 & 0.043 & 5.5 & & 3.7 \\
\hline 113 & $694 \mathrm{C}$ & 4C 3942 & $6 \times 05$ & 224.86 & 0.22 & 0.048 & 5.4 & & 4.6 \\
\hline 113 & $694 \mathrm{C}$ & 4 C 39 & $7 \mathrm{X}$ & 27. & 0.1 & 0.02 & 6.0 & & 7.2 \\
\hline 113 & $694 \mathrm{C}$ & $4 C 39$ & $7 \mathrm{X}$ & 2 & 0.0 & & 10.8 & & 4.0 \\
\hline 113 & $694 \mathrm{C}$ & $4 C 3$ & & & & & 1 & & 5.4 \\
\hline 113 & $694 \mathrm{C}$ & & & & & & & & 2.9 \\
\hline 113 & $694 \mathrm{C}$ & & & & & & & & .3 \\
\hline 113 & $694 \mathrm{C}$ & & & & & & & & 4.4 \\
\hline 113 & $694 \mathrm{C}$ & $4 \mathrm{C} 3$ & & & & & & & 4.2 \\
\hline 113 & $694 \mathrm{C}$ & $4 C^{2} 3$ & & & & & & & 3.0 \\
\hline 113 & $694 \mathrm{C}$ & 4C3953 & 9X01 & & & & 14 & & 3.1 \\
\hline 113 & $694 \mathrm{C}$ & 4C3954 & 9X01 & 247.15 & 0.24 & 0.055 & 5 & & 4.7 \\
\hline 113 & $694 \mathrm{C}$ & 4C3955 & 9X01 & 247.45 & 0.25 & 0.053 & 5.6 & & 5.0 \\
\hline 113 & $694 \mathrm{C}$ & $4 C 3956$ & $10 \times 01$ & 256.24 & 0.21 & 0.056 & 4.4 & -22 & 3.4 \\
\hline 113 & $694 \mathrm{C}$ & $4 C 3$ & $10 x$ & 2 & 0.69 & 0.10 & 7.0 & -2 & 4.3 \\
\hline 13 & $694 \mathrm{C}$ & & & & & 0.6 & 8.2 & & 4.1 \\
\hline 13 & $694 \mathrm{C}$ & & & & & & 12. & & 3.9 \\
\hline 13 & $694 \mathrm{C}$ & & & & & & & & 4.1 \\
\hline 13 & $694 \mathrm{C}$ & & & & & & & & 4.0 \\
\hline 113 & $694 \mathrm{C}$ & $4 \mathrm{C} 3$ & & & & & & & 5.1 \\
\hline 113 & $694 \mathrm{C}$ & $4 \mathrm{C} 3$ & & & & & & & 4.3 \\
\hline 113 & $694 \mathrm{C}$ & 4C3964 & $10 \mathrm{Xc}$ & & & & & & 4.1 \\
\hline 113 & $694 \mathrm{C}$ & 4C 3965 & $10 \mathrm{XCC}$ & 258.81 & 0.06 & 0.0 & 10.0 & & 4.0 \\
\hline 113 & $694 \mathrm{C}$ & 4C3966 & $11 \times 01$ & 265.65 & 0.58 & 0.090 & 7.5 & -2 & 4.2 \\
\hline 113 & $694 \mathrm{C}$ & 4C3967 & $11 \times 01$ & 265.85 & 0.29 & 0.041 & 8.3 & -23.2 & 3.8 \\
\hline 113 & $694 \mathrm{C}$ & 4C3968 & $11 x$ & 266.22 & 1.01 & 0.091 & 12.9 & -23 & 3.6 \\
\hline 13 & $694 \mathrm{C}$ & & & 266. & 0.40 & 0.0 & 2.0 & -2 & 2.3 \\
\hline 13 & $694 \mathrm{C}$ & & & & 0.4 & & 3.2 & & 3.0 \\
\hline 13 & $694 \mathrm{C}$ & & & & & & & & 3.5 \\
\hline 13 & $694 \mathrm{C}$ & & & & & & & & 3.6 \\
\hline 113 & & & & & & & & & 3.8 \\
\hline 113 & $694 \mathrm{C}$ & 4 C 39 & & & & & & & .5 \\
\hline 113 & $694 \mathrm{C}$ & $4 C 3976$ & $11 \times 0$ & 268. & & & & & 3.3 \\
\hline 113 & $694 \mathrm{C}$ & 4C3977 & $12 \times 01$ & 276.09 & 0.20 & 0.0 & 7 & & \\
\hline 113 & $694 \mathrm{C}$ & $4 C 3978$ & $12 \times 01$ & 276.44 & 0.18 & 0.02 & 8.0 & -2 & 4.9 \\
\hline 113 & $694 \mathrm{C}$ & 4C3979 & $12 \times 01$ & 276.71 & 0.18 & 0.013 & 16.0 & -2 & 3.4 \\
\hline 113 & $694 \mathrm{C}$ & 4C3980 & $13 \mathrm{X}$ & 285.43 & 0.55 & 0.03 & 16.5 & -23 & 3.3 \\
\hline 113 & $694 \mathrm{C}$ & 4C39 & $13 x$ & 286.05 & 0.9 & 0.0 & 16.1 & -23 & 3.1 \\
\hline 13 & $694 \mathrm{C}$ & & & & 0.9 & & 13.7 & -2 & 3.0 \\
\hline 13 & 6 & & & & 0.3 & & 2.8 & & 4.2 \\
\hline 13 & & & & & & & & & 5.4 \\
\hline 13 & & & & & & & & & 4.3 \\
\hline 113 & 694 & & & & & & & & 4.6 \\
\hline 113 & $694 \mathrm{C}$ & 4C3989 & $14 \times 02$ & 296.84 & 0.36 & & & & .8 \\
\hline 113 & $694 \mathrm{C}$ & 4C3990 & $14 \times 02$ & 297.16 & 0.23 & 0.030 & 9.0 & & 5.2 \\
\hline 113 & $694 \mathrm{C}$ & 4C3991 & $14 \times 02$ & 297.40 & 0.42 & 0.0 & 7.1 & & 4.3 \\
\hline 113 & $694 \mathrm{C}$ & $4 C 3992$ & $14 \times 03$ & 298.03 & 0.23 & 0.02 & 9.6 & -2 & 4.9 \\
\hline 113 & $694 \mathrm{C}$ & 4C3993 & $14 \times 03$ & 298.34 & 0.44 & 0.0 & 5.7 & -23.1 & 4.5 \\
\hline 113 & $694 \mathrm{C}$ & 4C 399 & $14 X$ & 298.6 & 0.4 & 0.0 & 9.5 & -2 & 4.7 \\
\hline 13 & $694 \mathrm{C}$ & & & 298. & 0.2 & & 9.6 & & 4.7 \\
\hline 13 & $694 \mathrm{C}$ & & & & 0.0 & & 2.8 & & 4.9 \\
\hline 13 & 6 & & & & 0.3 & & 8.9 & & 4.3 \\
\hline 1 & & & & & & & 10.7 & & 4.2 \\
\hline 113 & & & & & & & & & 4.5 \\
\hline 113 & $694 \mathrm{C}$ & & & & 0.32 & & 9.4 & & 4.7 \\
\hline 113 & $694 \mathrm{C}$ & & & & & & 8.9 & & \\
\hline 113 & $694 \mathrm{C}$ & $4 C 400$ & & & & & 6.8 & & 3.8 \\
\hline 113 & $694 \mathrm{C}$ & $4 C 4003$ & $14 \times 06$ & & & & 6.0 & & 3.9 \\
\hline 113 & $694 \mathrm{C}$ & $4 C 4004$ & $14 \times 06$ & 302.84 & 0.29 & 0.030 & 11.1 & & 3.1 \\
\hline 113 & $694 \mathrm{C}$ & $4 C 4005$ & $14 \times 06$ & 303.16 & 0.27 & 0.020 & 15.5 & & 2.9 \\
\hline 113 & $694 \mathrm{C}$ & 4C4006 & $14 \times 06$ & 303.40 & 0.34 & 0.028 & 14.1 & & 4.6 \\
\hline & & $4 C 4007$ & & & & & & & \\
\hline
\end{tabular}

Table 1 (continued).

\begin{tabular}{rccccccccc}
\hline Leg & Hole & Mun\# & Core & Depth & $\% \mathrm{C}$ & $\% \mathrm{~N}$ & $\mathrm{C} / \mathrm{N}$ & $\delta^{13} \mathrm{C}$ & $\delta^{15} \mathrm{~N}$ \\
\hline 113 & $694 \mathrm{C}$ & $4 \mathrm{C} 4008$ & $15 \times 01$ & 304.64 & 0.52 & 0.073 & 8.3 & -23.0 & 3.2 \\
113 & $694 \mathrm{C}$ & $4 \mathrm{C} 4009$ & $15 \times 01$ & 304.96 & 0.82 & 0.080 & 12.0 & -23.4 & 3.8 \\
113 & $694 \mathrm{C}$ & $4 \mathrm{C} 4010$ & $15 \times 01$ & 305.27 & 0.70 & 0.059 & 13.9 & -23.4 & 4.0 \\
113 & $694 \mathrm{C}$ & $4 \mathrm{C} 4011$ & $15 \times 01$ & 305.51 & 1.16 & 0.113 & 11.9 & -23.5 & 3.3 \\
113 & $694 \mathrm{C}$ & $4 \mathrm{C} 4012$ & $15 \times 02$ & 306.14 & 0.57 & 0.073 & 9.1 & -23.3 & 2.9 \\
113 & $694 \mathrm{C}$ & $4 \mathrm{C} 4013$ & $15 \times 02$ & 306.43 & 0.13 & 0.027 & 5.8 & -22.4 & 3.2 \\
113 & $694 \mathrm{C}$ & $4 \mathrm{C} 4014$ & $15 \times C C$ & 306.88 & 0.11 & 0.038 & 3.4 & -23.1 & 3.7 \\
113 & $694 \mathrm{C}$ & $4 \mathrm{C} 4015$ & $19 \mathrm{X} 01$ & 343.41 & 0.32 & 0.073 & 5.1 & -22.6 & 3.5 \\
113 & $694 \mathrm{C}$ & $4 \mathrm{C} 4016$ & $19 \mathrm{X} 01$ & 343.72 & 0.17 & 0.065 & 3.0 & -23.5 & 4.1 \\
113 & $694 \mathrm{C}$ & $4 \mathrm{G} 4017$ & $19 \mathrm{X} 01$ & 343.95 & 0.20 & 0.045 & 5.2 & -23.4 & 5.6 \\
113 & $694 \mathrm{C}$ & $4 \mathrm{C} 4018$ & $19 \mathrm{X} 01$ & 344.21 & 0.16 & 0.043 & 4.3 & -22.9 & 5.7 \\
113 & $694 \mathrm{C}$ & $4 \mathrm{C} 4019$ & $19 \mathrm{X} 01$ & 344.40 & 0.27 & 0.073 & 4.3 & -23.6 & 5.5 \\
113 & $694 \mathrm{C}$ & $4 \mathrm{C} 4020$ & $19 \mathrm{X} 02$ & 344.84 & 0.73 & 0.070 & 12.2 & -23.2 & 4.8 \\
\hline
\end{tabular}

continental slope, and have estimated maximum sedimentation rates of $180 \mathrm{~m} / \mathrm{m} . \mathrm{y}$. over a $500,000 \mathrm{yr}$ period.

Organic carbon and total nitrogen significantly correlate $\left(\mathrm{R}^{2}\right.$ $=0.50$ ) as does $\mathrm{C} / \mathrm{N}$ with $\% \mathrm{C}$ and ${ }^{13} \mathrm{C}$. The other parameters measured show no correlation at the 0.95 level.

\section{DISCUSSION}

With low sedimentation rates, as described above, preservation of organic matter is generally poor. In oxygenated waters, decreased sedimentation rates indicate enhanced time in the water column, and the greater likelihood of organic decomposition or alteration taking place (Muller and Suess, 1979; Muller et al., 1983). In biogenic sediments, productivity may be the principal control of the organic content of a sediment. Despite high abundances of microfossils in these sediments, the total organic loading associated with the productivity has not been preserved. These results would indicate that the rate of productivity over the sampling resolution time span (a minimum of 5,000 yr) was not high, and that what little material was produced, was recycled either in the water column or in the surficial sediments. $\mathrm{Cu}$ riously, the total nitrogen in these sediments is high, often equal to or greater than the organic carbon loading, and is likely caused by the bonding of ammonium to the sediment particles, resulting in $\mathrm{C} / \mathrm{N}$ near unity (Muller, 1977). This ammonium must have originated from organic production in the original water column and, in that sense, preserves evidence of a production rate greater than that seen in the organic carbon. Isotopic alteration may occur with diagenesis of the organic matter (Handa et al., 1972; Ittekkot et al., 1982; Entzeroth, 1982). In general, carbon isotopic compositions of organics in clastic sediments have been observed to be slightly $(1 \% 0)$ more enriched than overlying suspended particulate material. Particulate nitrogen isotopic compositions may be altered significantly during increased recycling or diagenesis (Altabet and McCarthy, 1986; Macko, 1983; 1989; Macko et al. 1986). The fact that $\% \mathrm{C}$ and $\% \mathrm{~N}$ correlate significantly for all sites appears to indicate a mechanism maintaining a related preservation of the two elements. It is important to note, however, that not only in organic content, but also in isotopic compositions, inconsistencies between compositions of carbon and nitrogen may simply be the result of different origins for the majority of that element (Mayer et al., 1988).

The organic data indicate changes in the types and quantity of production occurring in the water column, and suggest an increased intensity in the recycling of nitrogen since the Pleistocene. These results give support for present models on the development of the Antarctic ice-sheet and cooling of the waters surrounding the continent (Barker, Kennett, et al., 1988). An important consideration in the evaluation of the isotopic signatures in such a model is the observation that present day particulate organic material, which was collected in sediment traps si- 

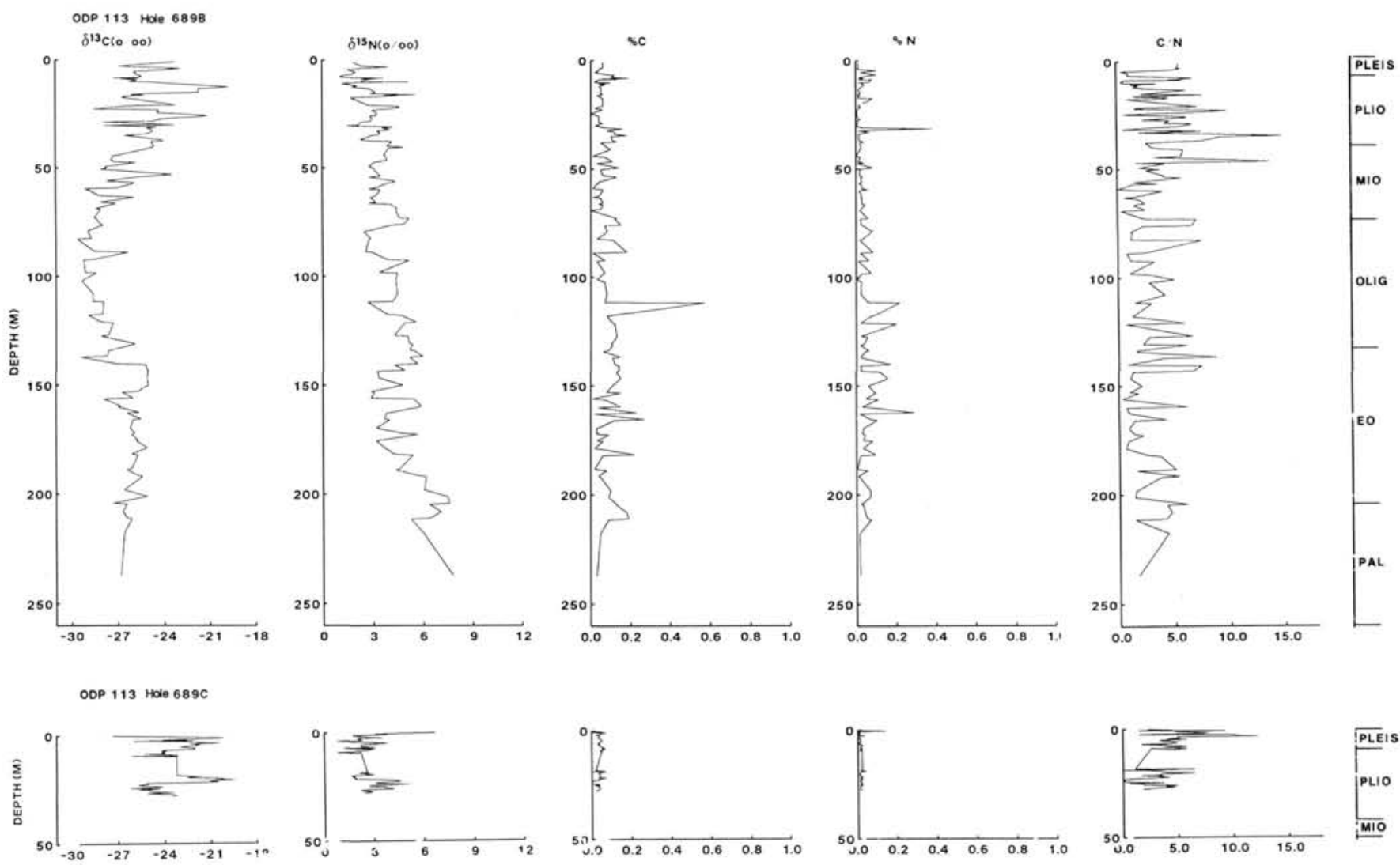

ODP 113 Hole 6890
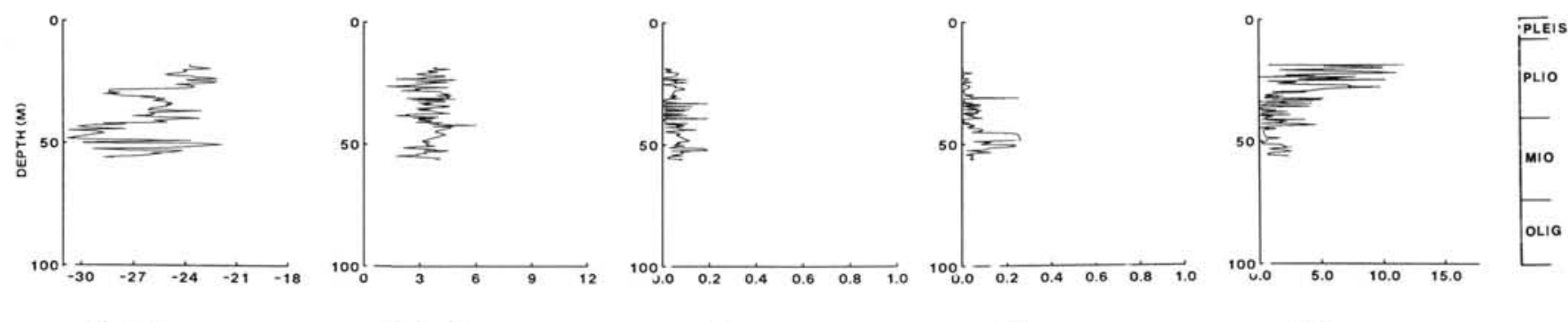

$$
\delta^{13} \mathrm{C}(0 / 00)
$$

Figure 2. Site 689 individual core results: $\delta^{13} \mathrm{C}, \delta^{15} \mathrm{~N}$, \%organic $\mathrm{C}, \%$ total $\mathrm{N}$, and $\mathrm{C} / \mathrm{N}$ vs. depth.

multaneously to the Leg 113 drilling operation, is depleted in both $\delta^{13} \mathrm{C}$ and $\delta^{15} \mathrm{~N}$, relative to most marine sediments (Biggs et al., 1988). These depletions in the sediment trap samples are able to be associated with fresh, organic rich material and are likely the result of isotopic fractionations by the primary producers. Isotopic compositions similar to these modern values were seen in the erratic spikes of Miocene age sediments at Site 693; these sediments appear to be rich in diatoms, from smear slide analyses (Barker, Kennett, et al. 1988). Such depleted compositions appear to be an indicator of primary production, specifically diatoms, in nutrient rich, cooler waters. Such depleted signatures are common at Sites 689 and 690 in the Oligocene. It is believed that these depletions in isotopic signatures, from more enriched values of the Eocene and Paleocene, are an indication of the cooling of the waters, and as these sites are removed from continental influences, of the waters surrounding Antarctica in general. The fact that the fossils and the isotopes only occasionally show these anomalous compositions is an indication that the events which caused them in the Miocene were erratic and very isolated, perhaps like present-day polynya in the sea ice. Also notable is the fact that carbon and nitrogen isotopic compositions dramatically increase from the middle Miocene onwards at Sites 689 and 690 . As noted above, such enrichments have been observed in modern settings associated with enhanced organic recycling. A further observation of the nitrogen isotopic changes at Site 690 is that a depletion occurs during the Pliocene, prior to the most enriched values of the Pleistocene. Those enriched values are not seen in the condensed Pleistocene section of Site 689 , and could indicate that the section is not as complete as that at Site 690 . A clear depletion of about $2 \% 0$ is also seen in carbon at Site 690 in the Pleistocene, which is not seen in the samples from Site 689. Isotopic compositions of the samples from Site 694 are similar to other reports of colder water carbon productivity; few nitrogen isotopic compositions exist for high latitude sediments. The values observed at Site 694 are similar to those seen in Recent sediments from the Bransfield Strait (Silfer et al., in press) and on the continental shelf of the modern Canadian Arctic Ocean. Since much of the sedi- 

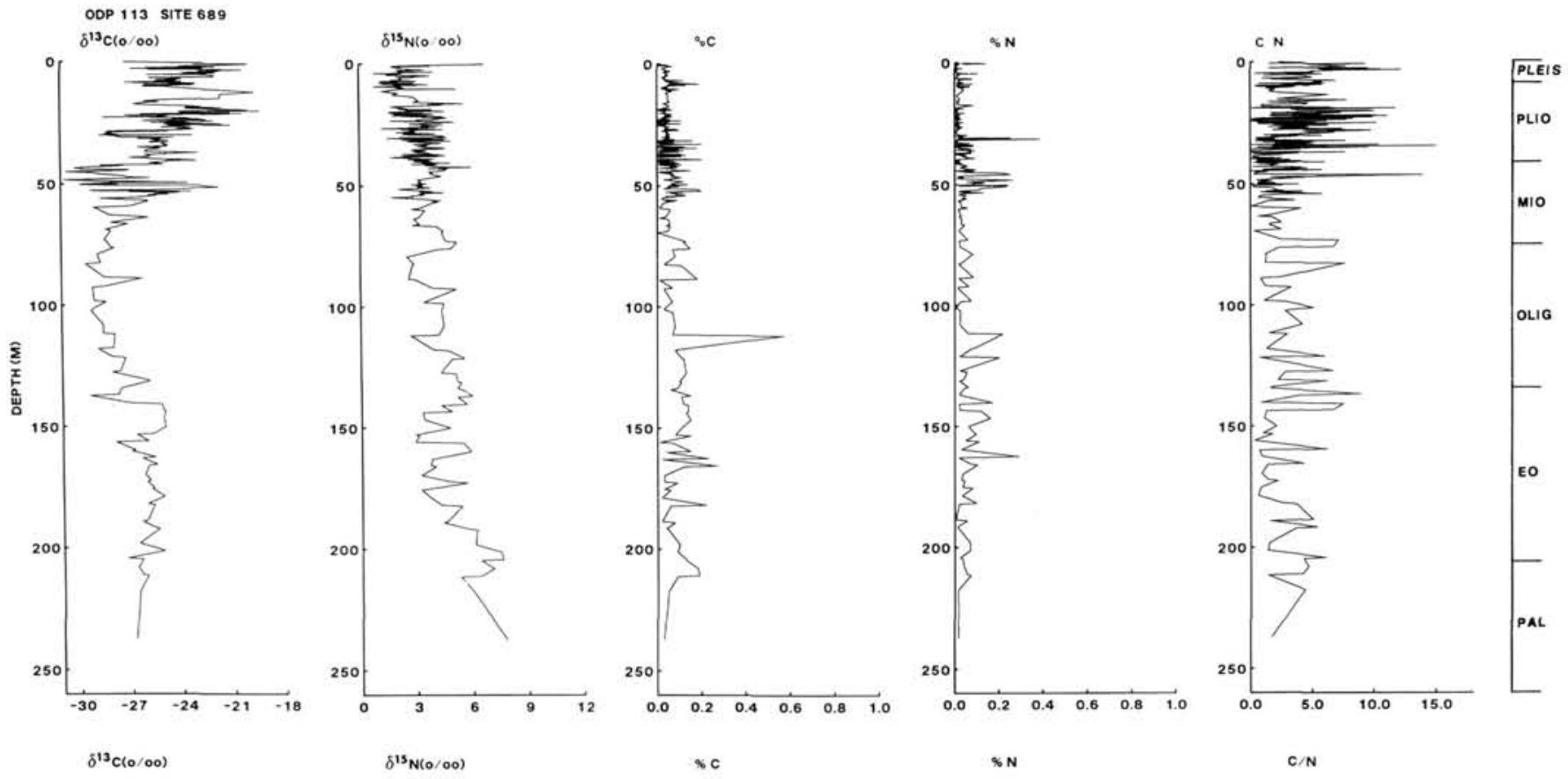

Figure 3. Site 689 composite core results: $\delta^{13} \mathrm{C}, \delta^{15} \mathrm{~N}$, \%organic $\mathrm{C}, \%$ total $\mathrm{N}$, and $\mathrm{C} / \mathrm{N}$ vs. depth.
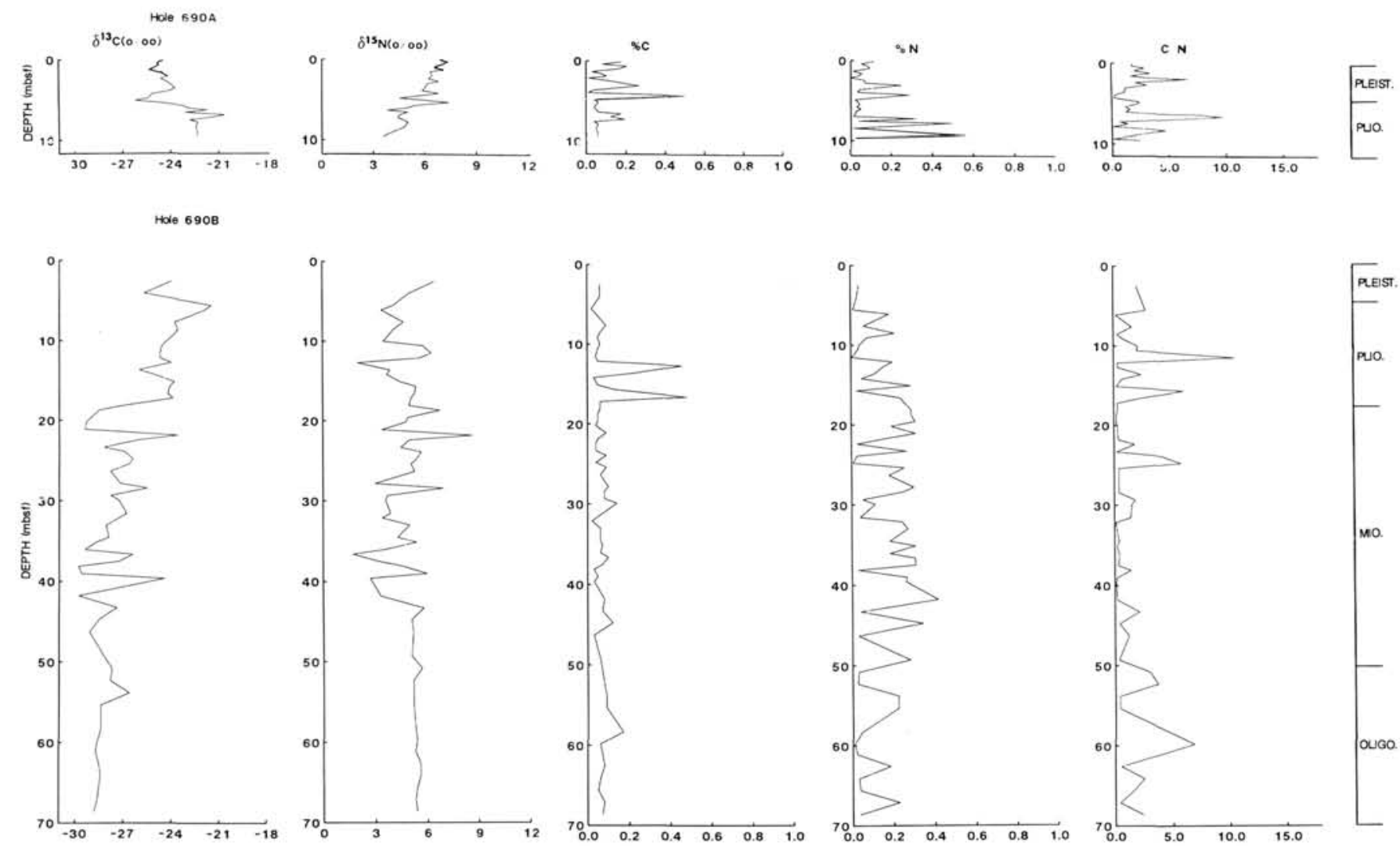

$\delta^{13} \mathrm{C}(0 / 00)$

$\delta^{15} \mathrm{~N}(0 / 00)$

sc

$\approx N$

$\mathrm{C} / \mathrm{N}$

Figure 4. Site 690 individual core results: $\delta^{13} \mathrm{C}, \delta^{15} \mathrm{~N}, \%$ organic $\mathrm{C}, \%$ total $\mathrm{N}$, and $\mathrm{C} / \mathrm{N}$ vs. depth. 

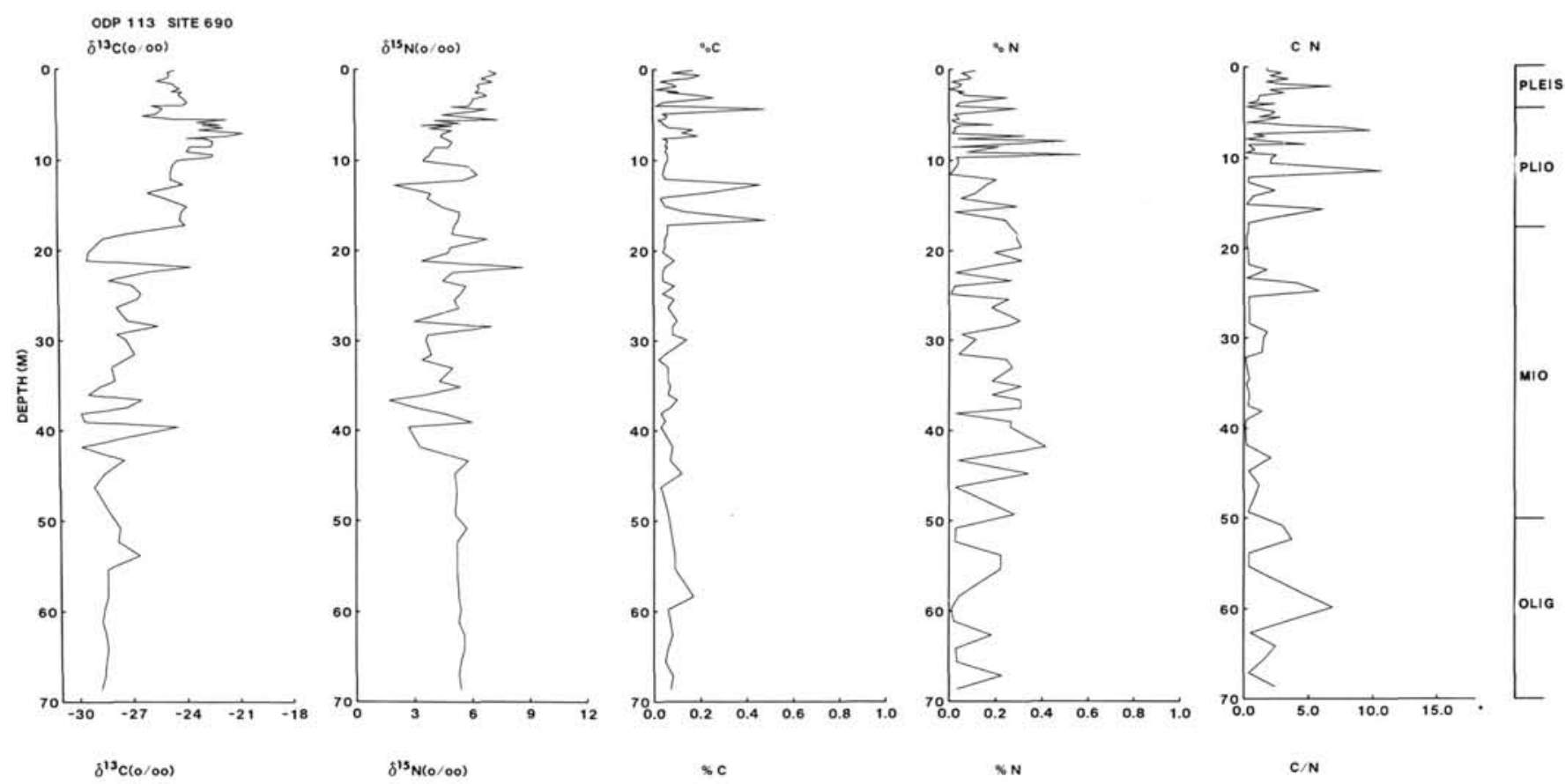

Figure 5. Site 690 composite core results: $\delta^{13} \mathrm{C}, \delta^{15} \mathrm{~N}, \%$ organic $\mathrm{C}, \%$ total $\mathrm{N}$, and $\mathrm{C} / \mathrm{N}$ vs. depth.

mentation at this site is the result of instabilities from the adjacent slope, these results suggest that the Site 694 sediments show compositions typical of ocean systems which are similar to those environments, and which were originally deposited on the slope and subsequently transported to the abyssal plain. The heightened organic composition of these Miocene sediments suggests higher production and preservation on the slope, perhaps prior to the Miocene events.

Finally, at Site 693 there is a major event, which is perhaps related to the progressive growth or the stabilization of the West Antarctic ice-sheet. At a depth of approximately $100 \mathrm{~m}$, all indicators of the organics in the present study show this event, which is estimated to be about $5 \mathrm{Ma}$. It is suggested that with stabilization of the ice-sheet, and less loss of sediment, through ice rafting or instability, the waters below the sea-ice saw increased recycling of carbon and nitrogen with enhanced binding of ammonium to sediment particles, and diminished carbon preservation. As a result the nitrogen isotopic signatures are somewhat enriched in the Pliocene and show the most enrichment in the Pleistocene.

\section{SUMMARY}

These preliminary data on the organic compositions and analyses of carbon and nitrogen isotopic compositions of sediments from the Weddell Sea indicate:

1. Changes in the organic material preserved in the sediments record events in the waters surrounding Antarctica which may be attributed to the cooling of those waters and glacial advance.

2. An event related to the progressive growth or stabilization of the West Antarctic ice-sheet is recorded at approximately 5 Ma.

3. With increases in the ice cover and its permanency, intensification in the recycling of organics is observed.

4. Overall poor preservation of organic matter is seen for most sediments and this is probably the result of slow sedimentation rates; an oxygenated water column, which was corrosive to organic matter; and an overall low productivity. Much of the nitrogen preserved in the sediments is in the form of ammonium bound to sediment particles.

\section{ACKNOWLEDGMENTS}

Appreciation is expressed to the crew and scientific party of ODP Leg 113 for the collection of these samples. This research was made possible by research grants from NSERC (individual operating grant A2644) and the Petroleum Research Fund, administered by the American Chemical Society (14805-AC2). The laboratory assistance of K. Pulchan, W. Gallant, A. Collins, P. Harrigan, N. Ostrom, and A. McIlraith is greatly appreciated. The comments by three reviewers strengthened the final manuscript.

\section{REFERENCES}

Altabet, M. A., and McCarthy, J. J., 1986. Vertical patterns in ${ }^{15} \mathrm{~N}$ natural abundance in PON from the surface waters of warm-core rings. J. Mar. Res., 44:185-201.

Barker, P. F., Kennett, J. P., et al., 1988. Proc. ODP, Init. Repts., 113: College Station, TX, (Ocean Drilling Program).

Biggs, D. C., Berkowitz, S. P., Altabet, M. A., Bidigare, R. R., DeMaster, D. J., Dunbar, R. B., Leventer, A., Macko, S. A., Nittrouer, C. A., and Ondrusek, M. E., 1988. A cooperative study of upper ocean particulate fluxes in the Weddell Sea. In Barker, P. F., Kennett, J. P., et al., Proc. ODP, Init. Repts., 113: College Station, TX. (Ocean Drilling Program), 77-85.

Degens, E. T. 1969. Biogeochemistry of stable carbon isotopes. In Eglinton, G., and Murphy M.T.J., (Eds.), Organic Geochemistry: New York (Springer-Verlag), 304-329.

Degens, E. T., Gaillard, R.R.L., Sackett, W. M., and Hellebust, J. A. 1968. Metabolic fractionation of carbon isotopes in marine plankton-I. Temperature and respiration experiments. Deep-Sea Res., 15:1-9.

Eckelmann, W. R., Broecker, W. S., Whitlock, D. W., and Allsup, J. R., 1962. Implications of carbon isotopic composition of total organic carbon of some recent sediments and ancient oils. AAPG Bull., 46: 699-704.

Entzeroth, L. C. 1982. Particulate matter and organic sedimentation on the continental shelf and slope of the northwest Gulf of Mexico [Ph.D. dissert.], Univ. Texas, Austin. 
Fontugne, M. R., and Duplessy, J. C., 1981. Organic carbon isotopic fractionation by marine plankton in the temperature range $-1^{\circ}$ to $31^{\circ} \mathrm{C}$. Oceanol. Acta, 4:85-90.

1978. Carbon isotope ratio of marine plankton related to surface water masses. Earth Planet, Sci. Lett. 41:365-371.

Gearing, J. N., Gearing, P. J., Rudnick, D. T., Requejo, A. G. and Hutchings, M. J., 1984. Isotopic variability of organic carbon in a phytoplankton-based, temperate estuary. Geochim. Cosmochim. Acta, 48:1089-1098.

Gearing, P., Plucker, P. F., and Parker, P. L., 1977. Organic carbon stable isotope ratios of continental margin sediments. Mar. Chem., 5: 251-256.

Handa, N., Yunagi, K., and Motsunaga, S. 1972. Distribution of detrital materials in the western Pacific Ocean and their biochemical nature. Mem. Ist. Ital. Idrobiol., 29 suppl.:53-71.

Hedges, J. I., and Parker, P. L., 1976. Land derived organic matter in surface sediments from the Gulf of Mexico. Geochim. Cosmochim. Acta, 40:1019-1029.

Ittekkot, V., Degens, E. T. and Brockman, U., 1982. Monosaccharide composition of acid-hydrolyzable carbohydrates in particulate matter during a plankton bloom. Limnol. Oceanog. 27:770-776.

Macko, S. A. 1981. Stable nitrogen isotope ratios as tracers of organic geochemical processes. [Ph.D. dissert.], Univ. Texas, Austin.

1983. Sources of organic nitrogen in Mid-Atlantic coastal bays and continental shelf sediments of the United States: isotopic evidence, Carnegie Institute Washington Year Book 82:390-394. 1989. Stable isotope organic geochemistry of sediments from the Labrador Sea (Sites 646 and 647) and Baffin Bay (Site 645), ODP Leg 105. In Srivastava, S. P., Arthur, M., et al., Proc. ODP, Sci. Results, 105: College Station, TX (Ocean Drilling Program), 209-231.

Macko, S. A., Aksu, A. and Mudie, P., 1986. Paleoclimatic history of the Nansen Sound area, Arctic Ocean. Geol. Soc. Amer. Abstr. 6: 678.

Macko, S. A., Entzeroth, L. and Parker, P. L., 1984. Regional differences in nitrogen and carbon isotopes on the continental shelf of the Gulf of Mexico. Naturwissenschaften, 71:374-375.

Macko, S. A., Estep, M.L.F., Hare, P. E. and Hoering, T. C. 1987a. Isotopic fractionation of nitrogen and carbon in the synthesis of amino acids by microorganisms. Chem. Geol., 65:79-92.

Macko, S. A., Pulchan, K. and Ivany, D. E. 1987b. Organic geochemistry of Baffin Island fjords. Sedimentology of Arctic fjords experiment. Geol. Surv. Can., Open File Report 1589: 13:1-34.

Mayer, L. M., Macko, S. A. and Cammen, L. 1988. Provenance, concentrations, and nature of sedimentary organic nitrogen in the Gulf of Maine. Mar. Chem., 25:291-304.

Muller, P. J., 1977. C/N ratios in Pacific deep sea sediments: effect of inorganic ammonium and organic nitrogen compounds sorbed by clays. Geochim. Cosmochim. Acta, 41:765-776.

Muller, P., Erlenkeuser, H., and Grafenstein, R. V., 1983. Glacial-interglacial cycles in oceanic productivity inferred from organic carbon contents in eastern North Atlantic sediment cores. In Thiede, J., and
Suess, E., (Eds). Coastal Upwelling, Part B: New York (Plenum Press), 365-398.

Muller, P. J., and Suess, E., 1979. Productivity, sedimentation rate, and sedimentary carbon content in the oceans. 1. Organic carbon preservation. Deep-Sea Res., 26A:1347-1362.

Newman, J. W., Parker, P. L., and Behrens, E. W., 1973. Organic carbon isotope ratios in Quaternary cores from the Gulf of Mexico. Geochim. Cosmochim. Acta, 37:225-238.

Parker, P. L., Behrens, E. W., Calder, J. A., and Shultz, W., 1972. Stable carbon isotope ratio variations in the organic carbon from Gulf of Mexico sediments. Contrib. Mar. Sci., 16:139-147.

Peters, K. E., Sweeney, R. E. and Kaplan, I. R. 1978. Correlation of carbon and nitrogen stable isotope ratios in sedimentary organic matter. Limnol. Oceanogr., 23:598-604.

Rogers, M. A., van Hinte, J., and Sugden, J. G., 1972. Organic carbon ${ }^{13} \mathrm{C}$ values from Cretaceous, Tertiary and Quaternary marine sequences in the North Atlantic. In Laughton, A. S., Berggren, W. A., et al., Init. Repts. DSDP, 12: Washington (U.S. Govt. Printing Office), 1115-1126.

Sackett, W. M., 1986a. $\delta^{13} \mathrm{C}$ signatures of organic carbon in southern high latitude deep sea sediments; paleotemperature implications. Org. Geochem., 9:63-68.

1986b. Organic carbon in sediments underlying the Ross Ice Shelf. Org. Geochem., 9:135-138.

Sackett, W. M., Eadie, B. J. and Exner, M. E., 1974. Stable isotopic composition of organic carbon in recent Antarctic sediments. In Tissot, B. and Bienner, F. (Eds.) Adv. Org. Geochem., 661-671.

Sackett, W. M., Eckelmann, W. R., Bender, M. L. and Bé, A.W.H. 1965. Temperature dependence of carbon isotope compositions in marine plankton and sediments. Science, 148:235-237.

Sackett, W. M., and Thompson, R. R., 1963. Isotope organic carbon composition of Recent continental derived clastic sediments of Eastern Gulf Coast, Gulf of Mexico. AAPG Bull., 147:535-531.

Sigleo, A. C. and Macko, S. A., 1985. Stable isotope and amino acid composition of estuarine dissolved colloidal material. In Sigleo, A. C. and Hattori, A. (Eds.). Marine and estuarine geochemistry: Chelsea, MI. (Lewis Publishers Inc.), 30-46.

Silfer, J. A., Engel, M. H. and Macko, S. A. 1989. The effect of hydrothermal processes on the distribution and stereochemistry of amino acids in Recent Antarctic sediments. Applied Geochemistry.

Wada, E. 1980. Nitrogen isotope fractionation and its significance in biogeochemical processes occurring in marine environments. In Goldberg, E. D. and Horibe, Y. (Eds.), Isotope marine chemistry: Tokyo (Uchida Rokakuho Pub.), 375-398.

Zieman, J. C., Macko, S. A. and Mills, A. L. 1984. Role of seagrasses and mangroves in estuarine foodwebs: temporal and spatial changes in stable isotope composition and amino acid content during decomposition. Bull. Mar. Sci., 35:380-392.

Date of initial receipt: 27 March 1989

Date of acceptance: 11 October 1989

Ms 113B-190 

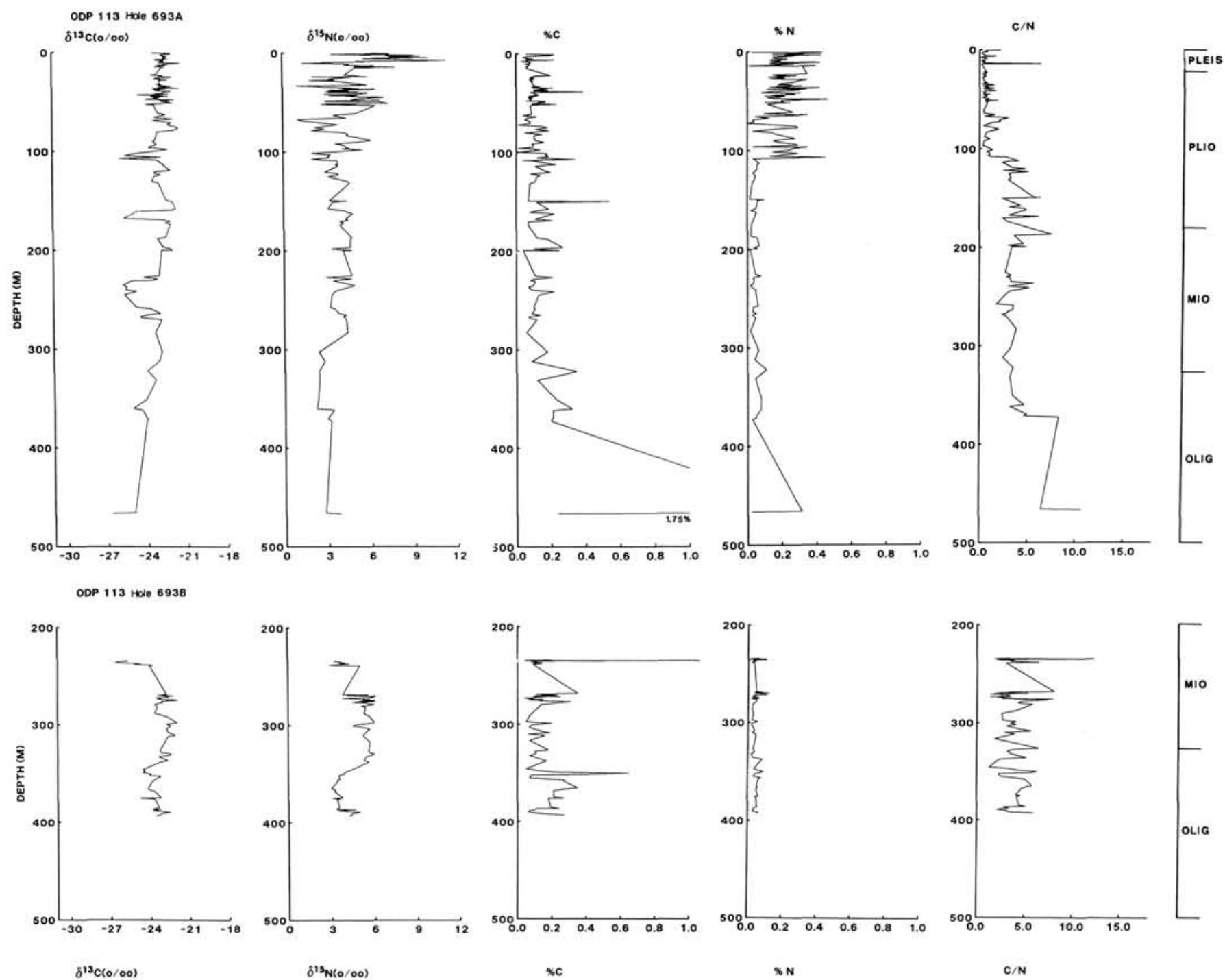

Figure 6. Site 693 individual core results: $\delta^{13} \mathrm{C}, \delta^{15} \mathrm{~N}, \%$ organic $\mathrm{C}, \%$ total $\mathrm{N}$, and $\mathrm{C} / \mathrm{N}$ vs. depth. 


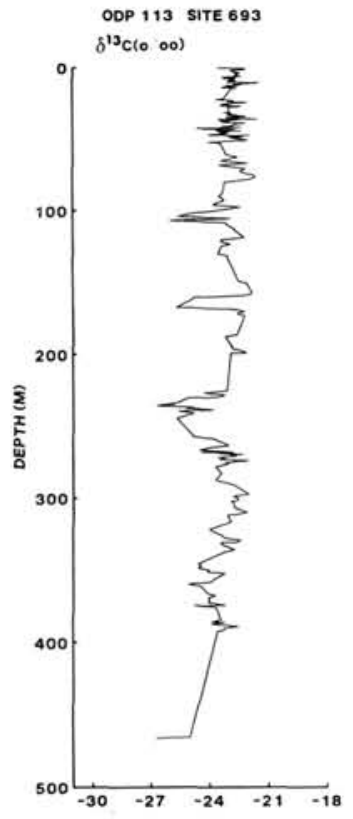

$\delta^{13} \mathrm{c}(0 / 00)$

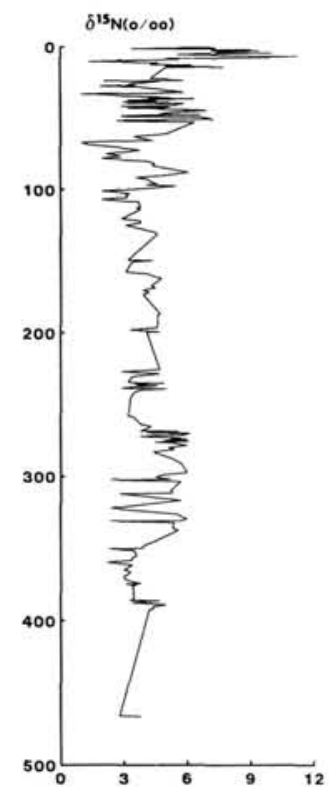

$\delta^{15} \mathrm{~N}(0 / 00)$

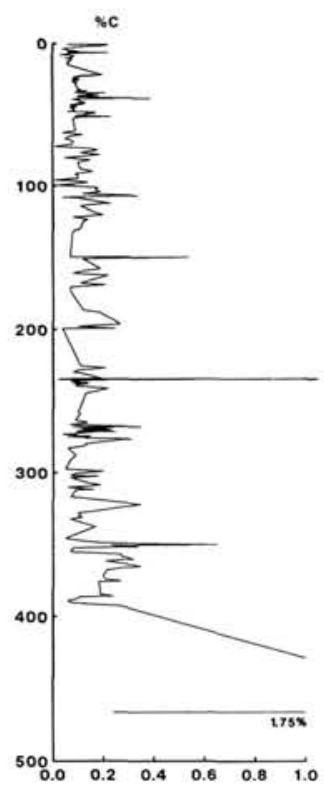

\& $\mathrm{C}$

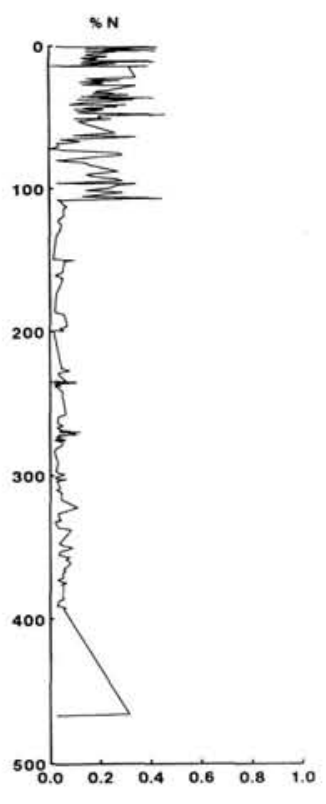

$\% \mathrm{~N}$

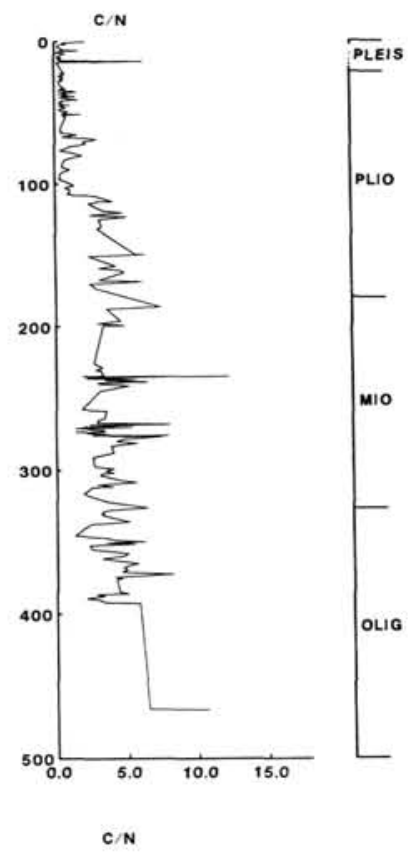

$\mathrm{C} / \mathrm{N}$

Figure 7. Site 693 composite core results: $\delta^{13} \mathrm{C}, \delta^{15} \mathrm{~N}$, \%organic $\mathrm{C}, \%$ total $\mathrm{N}$, and $\mathrm{C} / \mathrm{N}$ vs. depth. 

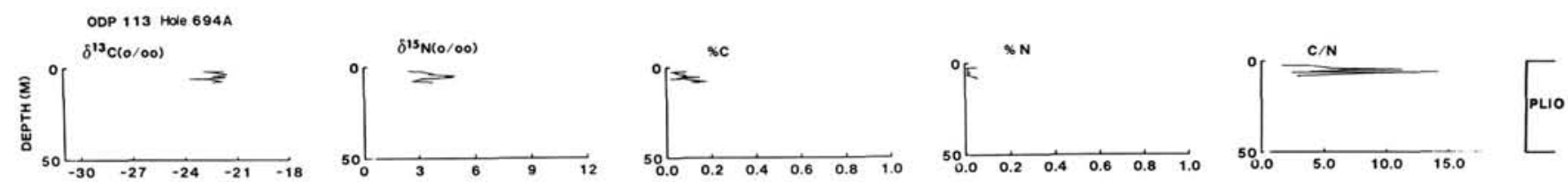

ODP 113 Hole 6948
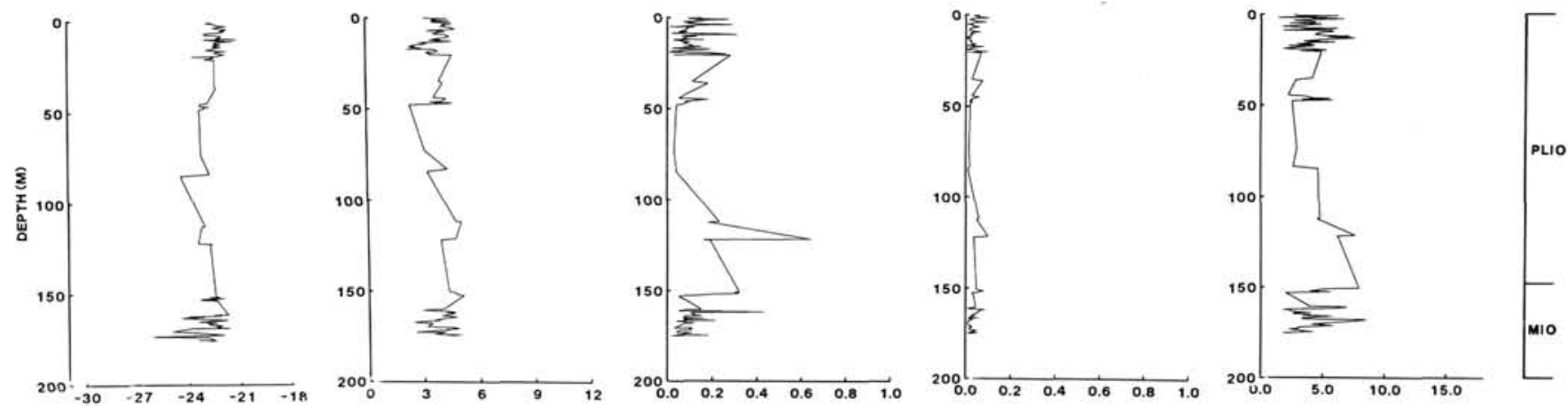

ODP 113 Hole $694 \mathrm{C}$
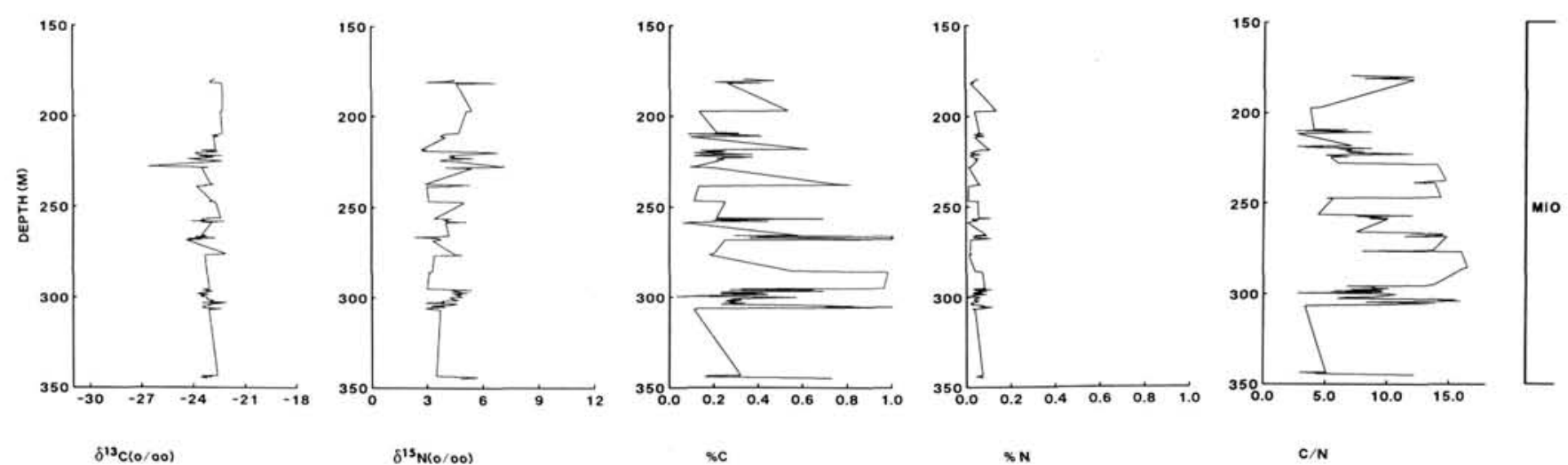

Figure 8. Site 694 individual core results: $\delta^{13} \mathrm{C}, \delta^{15} \mathrm{~N}, \%$ organic $\mathrm{C}, \%$ total $\mathrm{N}$, and $\mathrm{C} / \mathrm{N}$ vs. depth. 


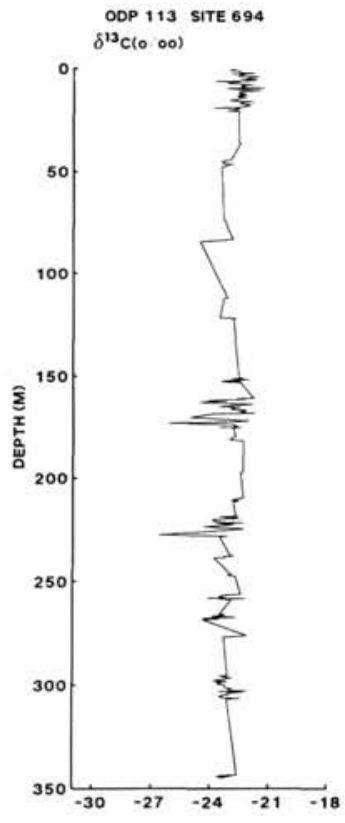

$\delta^{13} \mathrm{C}(0 / 00)$

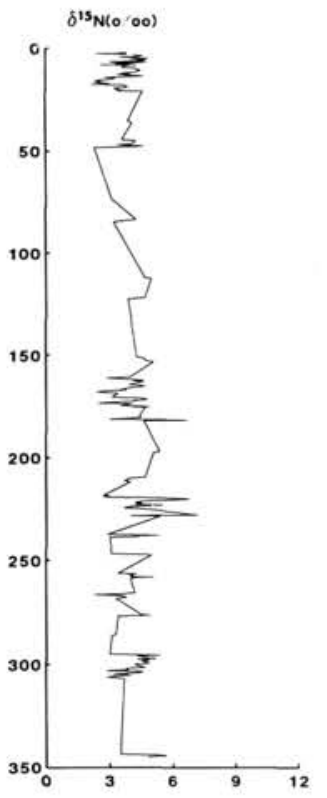

$\delta^{15} \mathrm{~N}(0 / 00)$

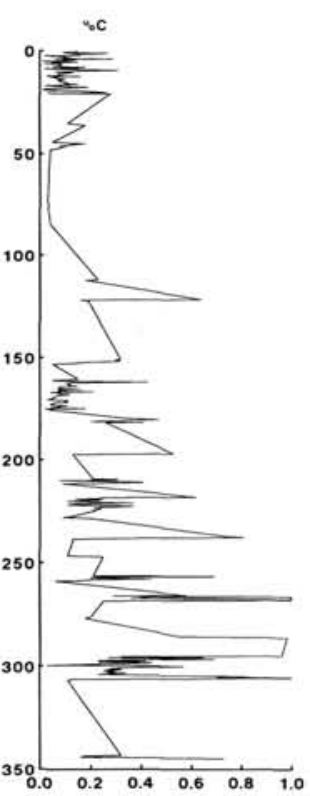

\%c

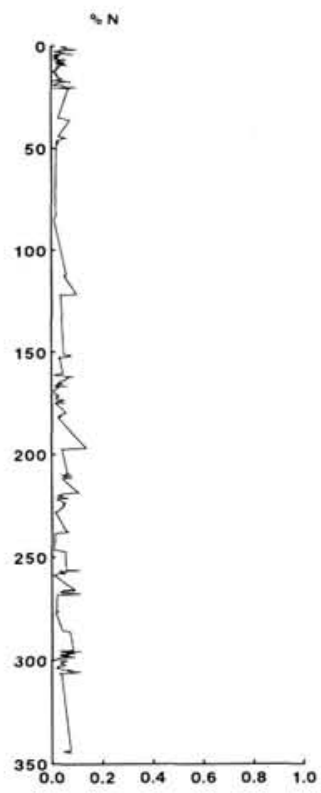

$\$ N$

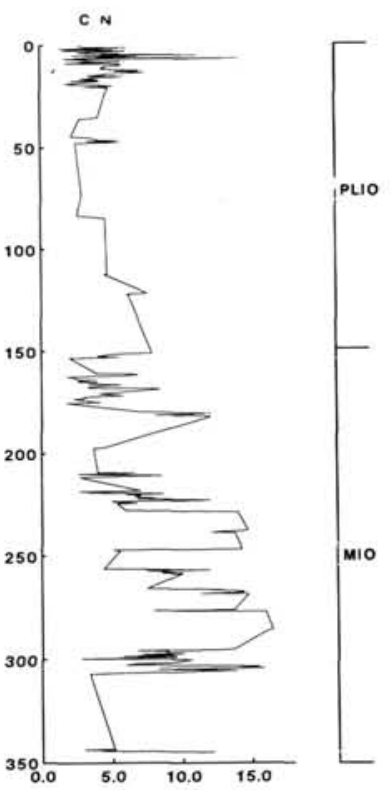

$\mathrm{C} / \mathrm{N}$

Figure 9. Site 694 composite core results: $\delta^{13} \mathrm{C}, \delta^{15} \mathrm{~N}, \%$ organic $\mathrm{C}, \%$ total $\mathrm{N}$, and $\mathrm{C} / \mathrm{N}$ vs. depth. 